\title{
Macropinocytosis activated by oncogenic Dbl enables specific targeted delivery of Tat/pDNA nano-complexes into ovarian cancer cells
}

This article was published in the following Dove Press journal: International Journal of Nanomedicine

\author{
Xiuran $\mathrm{Niu}^{1, *}$ \\ Zhihui Gaol,* \\ Shanshan Qi' \\ Linjia Su' \\ Nan Yang' \\ Xiuli Luan' \\ Jia $\mathrm{Li}^{\prime}$ \\ Qing Zhang² \\ Yingli $A^{3}$ \\ Sihe Zhang' \\ 'Department of Cell Biology, School \\ of Medicine, Nankai University, \\ Tianjin, People's Republic of China; \\ ${ }^{2}$ Department of Clinical Laboratory, \\ Cancer Hospital of Tianjin Medical \\ University, Tianjin, People's Republic \\ of China; ${ }^{3}$ State Key Laboratory of \\ Medicinal Chemical Biology and \\ Institute of Polymer Chemistry, \\ Nankai University, Tianjin, People's \\ Republic of China \\ *These authors contributed equally \\ to this work
}

\begin{abstract}
Background: Successful implementation of gene therapy heavily relies on efficiently delivering genetic materials and specific targeting into cells. Oncogene-driven endocytosis stimulates nutrient uptake and also develops an endocytosis-mediated defense against therapeutic agents. Cell-penetrating peptides, typically HIV-Tat, are well known for efficient delivery of nucleic acid drugs but lack targeting specificity. Various passive targeting strategies were pursued to enhance the tumor targeting efficiency; however, they are still limited by complicated cellular endocytosis routes and the heterogeneity of cancer types.
\end{abstract}

Methods: Tat/pDNA complexes were noncovalently compacted and their physiochemical properties were determined. The siRNA pool and pLV-RNAi-GFP lentivirus were used to knock down $d b l$ oncogene (originally isolated from diffuse B-cell lymphoma) expression, and its overexpression was performed by plasmid transient transfection. The cellular uptake of fluorescent ligands was quantified by confocal imaging and flow cytometry analysis. The transgene efficiency was determined by the Luciferase expression assay. Rho GTPase activation was checked by the GST-Rho GTPase-binding domain pull-down assay.

Results: pGL3 plasmid DNA was noncovalently compacted with the Tat peptide into nano-size complexes at high N/P ratios. Macropinocytosis, a clathrin- and caveolin-independent endocytosis process, was shown to contribute to the uptake of middle-sized $(\sim 600 \mathrm{~nm})$ Tat/pGL3 complexes. Cell-type-specific variation in macropinocytosis was essentially controlled by the action of the $\mathrm{Dbl}$ oncogene. Onco-Dbl presentation constantly induced a high level of macropinocytosis activity in ovarian cancer cells. Onco-Dbl overexpression hyperstimulated macropinocytosis enhancement in cells mainly through actin cytoskeleton reorganization mediated by the PH domain and Rac1 activation. The Dbl-driven Rho GTPase signaling collectively determined the cell-type-specific macropinocytosis phenotype.

Conclusion: Such an aspect can be exploited to selectively confer targeted delivery of Tat/ pDNA nano-complexes into ovarian cancer cells. Our work provides a novel alternative for targeted delivery of cell-penetrating peptide-based nucleic acid drugs into certain tumor types if specific endocytosis pathways are used.

Keywords: onco-Dbl, macropinocytosis, Rac1, Tat/pDNA complex, targeting delivery

\section{Introduction}

Successful implementation of gene therapy highly relies on the efficient delivery of therapeutic genes into target cells of certain tissue. Nonviral-based nanoparticles are more suitable for disease treatment due to their higher loading capacity, better biocompatibility, non-tumorigenicity, simplicity in preparation, and flexibility in use. ${ }^{1-3} \mathrm{How}-$ ever, they have relatively low transfection efficiency, and some of them have toxic side
Department of Cell Biology, School of Medicine, Nankai University, 94 Weijin Road, Nankai District, Tianjin 30007I. People's Republic of China

Tel +862223495226

Email sihezhang@nankai.edu.cn 
effects (eg, inducing hemagglutination by cationic liposomes/ polymers) when complexed with plasmid DNA (pDNA), ${ }^{4,5}$

Cell-penetrating peptides (CPPs) are well known for their efficient intracellular delivery of various biomolecules, including therapeutic genes incorporated in pDNA. Numbers of CPPs, typically HIV-Tat, have been used for nucleic acid drug delivery both in vitro and in vivo. ${ }^{2,3,6,7}$ There are two ways to deliver nucleic acids with CPPs, covalent conjugation and noncovalent complexation. While covalent conjugation forms well-defined entities which have desirable characteristic for drug design, the loss of biological activity after labor-intensive chemical modification limits this approach for clinical use. ${ }^{2,8}$ In contrast, the noncovalent strategy relies on the electrostatic interaction between positively charged CPPs and anionic nucleic acids, which leads to nano-size complex formation with increased serum stability. ${ }^{2,9,10}$ Furthermore, noncovalent complexation seems more suitable for large, negatively charged pDNA delivery because of easy handling and auto-release of the cargo into live cells., ${ }^{2,6,11}$

One shortcoming of CPP-based nucleic acid drugs delivery is the general lack of target specificity. Two controlled delivery strategies were devised to selectively target cancer cells. Active targeting by the addition of a binding moiety (antibody or tumor-homing peptide) ensures specific attachment of CPP-nucleic acid complexes to target molecules overexpressed on the cancer cell surface. ${ }^{3,12-14}$ However, this strategy has problems: 1) adding a targeting moiety will not eliminate the uptake ability of CPP cargos by normal cells, which often causes undesired side effects; ${ }^{3}$ 2) sometimes the delivery efficacy is lost or the internalization mode is altered when CPP cargos are coupled to the targeting moiety..$^{13}$ Passive targeting of CPP-based nucleic acid complexes is frequently pursued through changing properties (eg, enhanced permeability and retention effect) caused by a reaction with endogenous microenvironment factors (eg, pH, enzyme) at tumor sites. Although attaining encouraging results, these passive targeting practices are still limited by the spatial distribution of the tumor microenvironment. ${ }^{8,11,12,15,16}$ Therefore, novel approaches to enhance the targeting efficiency of CPP-delivered nucleic acid drugs specifically to tumors are urgent.

It has long been noticed that different cell lines use differed endocytosis pathways for Tat-pDNA complex uptake. The uptake of the Tat-pCMVL complex is mainly through caveolae-mediated endocytosis (CvME) in human liver cancer and hamster ovary cells. In contrast, under the same condition, no CvME of the Tat-pCMVL complex by monkey kidney epithelial cells was observed. ${ }^{15}$ Different uptake mechanisms of the Tat-pEGFPLuc complex were also observed among human cervix cancer cells, monkey kidney fibroblasts, and mouse embryonic fibroblasts. ${ }^{16}$ These celltype-specific variations in the endocytosis pathway seem to strongly depend on cell properties. In most cases, clathrinmediated endocytosis (CME) and CvME were frequently observed in the uptake of Tat-pDNA complexes. However, macropinocytosis has also been found in some cases to be the dominating pathway. ${ }^{17,18}$ Considering that macropinocytosis is a nonselective fluid phase endocytosis that can occur in all cell lines ${ }^{19-21}$ and that dominating macropinocytosis occurs in certain cell types implies that there might be some specific factors driving macropinocytosis enhancement. Such an aspect may be exploited to confer the selectivity for targeted delivery of Tat-pDNA complexes.

In the present study, we evaluated the endocytosis pathway of the Tat/pDNA nano-complex among tumor and non-tumor cells, using a combination of biochemical, pharmacological, and cell biology approaches. Our data revealed that medium-sized $(\sim 600 \mathrm{~nm})$ Tat/pDNA complexes follow a macropinocytosis route for uptake. The oncogenic protein $\mathrm{Dbl}$ (first discovered in human diffuse B-cell lymphoma), which is uniquely high-expressed in ovarian cancer cells, hyperstimulates constant macropinocytosis via the $\mathrm{PH}$ domain mainly mediated by Rac1 GTPase activation. This cell-typespecific Tat/pDNA macropinocytosis enhancement is largely through the actin cytoskeleton reorganization controlled by Dbl-triggered Rho GTPase signaling. Our work provides a novel alternative for targeted delivery of Tat/pDNA nanocomplexes specifically into ovarian cancer cells.

\section{Materials and methods}

\section{Cell culture, peptide, plasmids, antibodies, and reagents}

SKOV3, Cos-7, HeLa, 3T3, 293, HepG2, and 7721 cells were obtained from the Type Culture Collection of the Chinese Academy of Sciences (People's Republic of China). The pGL3 Luciferase Reporter plasmid (pGL3-control, E1741, was used for monitoring transfection efficiency) and the Luciferase assay kit (E1501) were obtained from Promega Corporation (Fitchburg, WI, USA). The HIV-Tat-derived peptide (GRKKRRQRRRPPQC-amide) was obtained from GL Biochem Ltd (Shanghai, People's Republic of China). The rabbit anti-Cdc42 (SC-87), anti-RhoA (SC-179), anti-Rac1 (SC-217), anti-Dbl pAb (SC-89), mouse anti-GST mAb (SC-138), and anti-a-tubulin (sc-5286) were obtained from Santa Cruz Biotechnology Inc. (Dallas, TX, USA). The rabbit anti-B-actin Ab (AB10001), anti-GFP Ab (D1100008), and nocodazole (A606391) were 
obtained from Sangon Biotech (Shanghai, People's Republic of China). The YOYO-1 (Y3601), Dextran-AF647 (D22914), Tfn-AF647 (T-23366), CTxB-AF647 (C-34778), goat anti-mouse IgG-AF488 (A-11001), anti-mouse IgG-AF647 (A32728), anti-rabbit IgG-AF488 (A-11070), 5-(N-ethyl-Nisopropyl) amiloride (E3111), Lipofectamine 3000 (L3000015), Lipofectamine ${ }^{\circledR}$ RNAiMax (13778075), and the BCA protein assay Kit (23225) were obtained from Thermo Fisher Scientific (Waltham, MA, USA). Rhodamine-phalloidin (PHDR1) was purchased from Cytoskeleton, Inc (Denver, CO, USA). The Rac1 inhibitor (55350), Cdc42 inhibitor (217708), ROCK inhibitor (SCM075), and PAK1 inhibitor (506101) were obtained from Merck (Darmstadt, Germany). Rhodamine B isothiocyanate-dextran (R9397), Cytochalasin D (C2618), Hochest33342 (B2261), polybrene (H9268), puromycin (P7255), and all fine grade chemicals were obtained from Sigma (Darmstadt, Germany).

\section{Preparation of tat/pDNA-YOYO-I complexes}

The pGL3 plasmid ( $35 \mu \mathrm{g} / \mathrm{mL}$ ) was labeled with YOYO-1 $\left(10^{-5} \mathrm{M}\right.$, in PBS) at a 1:5 ratio of dye molecule to DNA base pair $(\mathrm{d} / \mathrm{bp})$. The complexes were generated in $150 \mathrm{mM} \mathrm{NaCl}$ by drop-wise addition of Tat peptides to pGL3-YOYO-1 (30 minutes at room temperature). Different amounts of peptides were added to obtain N/P ratios (ie, the molar ratio of total free amino groups in the Tat peptide to total free phosphate groups in pDNA taken in solution) that ranged from 1 to 20 (Table S1).

\section{Electrophoretic mobility and retardation assay}

pGL3 plasmids labeled with/without YOYO-1 were electrophoresed on agarose gel (containing EB) at $100 \mathrm{~V}$ for 45 minutes to check the mobility difference. To evaluate the stabilities of complexes, Tat/pGL3 or Tat/pGL3-YOYO-1 complexes at different $\mathrm{N} / \mathrm{P}$ ratios were subjected to agarose electrophoresis to check the retardation effect.

\section{Fluorescent quenching assay}

Tat peptides were serially diluted in PBS corresponding to the indicated N/P ratios. Aliquots of pGL3-YOYO-1 (d/bp =1:5) were pipetted to either the Tat solution or PBS buffer, and mixed thoroughly. The sample fluorescence was measured in quadruples (Ex filter: 480 nm, Em filter: 515 nm, FLUOstar Omega). The DNA condensation was calculated as $\left(\mathrm{FL}_{\text {comp }}\right.$ $\left.\mathrm{FL}_{\mathrm{NDNA}}\right) \times 100$, where $\mathrm{FL}_{\text {comp }}$ and $\mathrm{FL}_{\mathrm{NDNA}}$ were peak fluorescent emissions of complexed and naked DNA, respectively.

\section{DNase I protection assay}

Tat/pGL3 or Tat/pGL3-YOYO-1 samples (diluted in $15 \mathrm{mM}$ $\mathrm{NaCl}$ ) were treated with DNase I ( 1 unit per $1 \mu \mathrm{g}$ DNA), and the mixtures were incubated at $37^{\circ} \mathrm{C}$ for 60 minutes. Samples were subjected to agarose electrophoresis analysis.

\section{Size and $\zeta$ potential measurement}

The particle sizes, either in PBS $(150 \mathrm{mM} \mathrm{NaCl})$ or in icDMEM (110 mM NaCl), were determined by dynamic light scattering, and $\zeta$ potentials were electrophoretically measured by ZETAPALS/BI-200SM (BROOKHAVEN). All data were collected over 2 minutes for each sample.

\section{Luciferase gene expression assay}

Tat/pGL3 complexes (10 $\mu \mathrm{g}$ of pDNA) diluted in $2.5 \mathrm{~mL}$ incomplete medium with/without corresponding drugs (Table S2) were added to $35 \mathrm{~mm}$ culture dishes (cell density: $2 \times 10^{4}$ per $\mathrm{cm}^{2}$ at $37^{\circ} \mathrm{C}$ or at $4^{\circ} \mathrm{C}$ for 4 hours of transfection. After changing to complete medium for an additional 38 hours growth, cells were lysed, debris-cleared, and aliquoted in triplicate for the Luciferase expression assay. The light emission was measured by integration over 10 seconds at $25^{\circ} \mathrm{C}$ (FLUOstar Omega). The activity (relative light units, RLU) was normalized for protein content.

\section{Dbl constructs for overexpression or knockdown}

Proto-Dbl, onco-Dbl, and their limited domain gene fragments (Figure 1A) were previously constructed and the GST-Nterminal cloned into $\mathrm{pEBG}$ plasmids for overexpression. ${ }^{22-24}$ Recombinant Dbl plasmids were transient transfected by Lipofectamine ${ }^{\circledR} 3000$ according to the manufacturer's instructions. Two kinds of Dbl knockdown approaches were used: the siRNA oligo pool (GenePharma) was used for short-term gene silencing in most uptake-checking assays. To observe the constant changes of the cellular skeleton, the GFP-tagged pLV-RNAi system (Biosettia) was used for long-term gene silencing (Table S3). The Dbl-shRNA lentivirus (in pLVH1-EF1 $\alpha$-GFP-Puro plasmid, SORT-B29) stably transduced Cos-7 and SKOV3 cell lines were similarly produced as previously described. ${ }^{25,26}$

\section{Uptake measurement by confocal imaging analysis}

The incubation conditions for endocytosis markers (TfnAF647, 15 minutes; Dextran-AF647/dextran-RITC, 1 hour; CTxB-AF647, 30 minutes) and Tat/pGL3-YOYO-1 complexes (10 $\mu \mathrm{g}$ pDNA/mL, 1 hour) were first optimized (Table S2). Transfected cells were incubated with fluorescent 
A

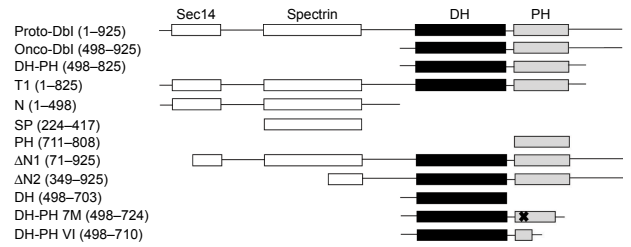

B

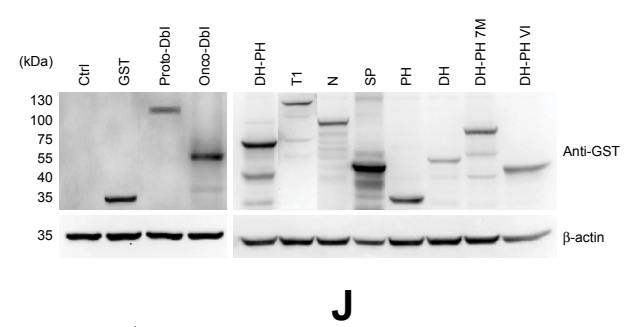

H

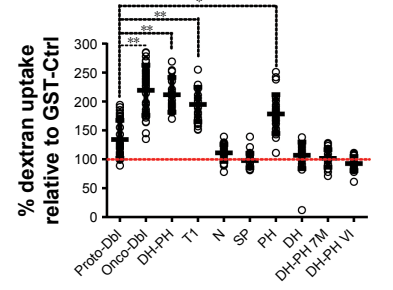

I

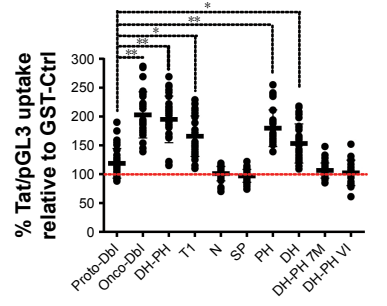

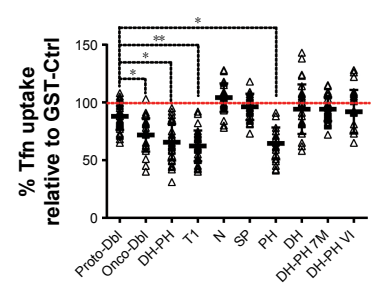

$\mathrm{K}$

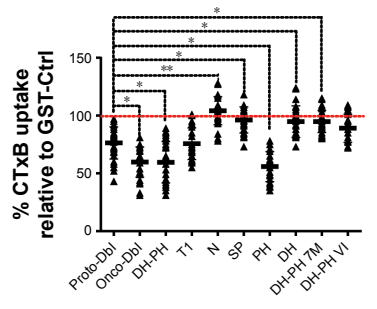

C
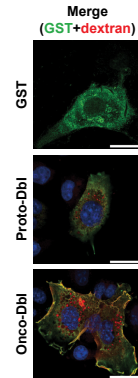

존
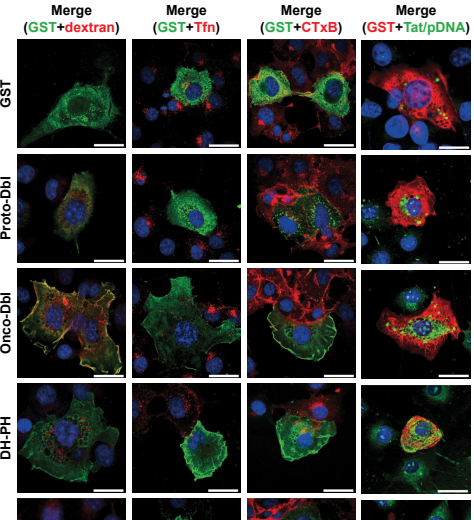

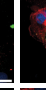
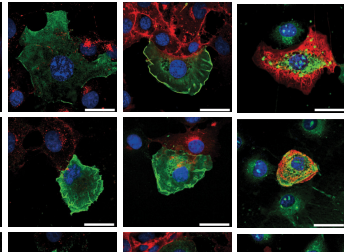

\section{E}
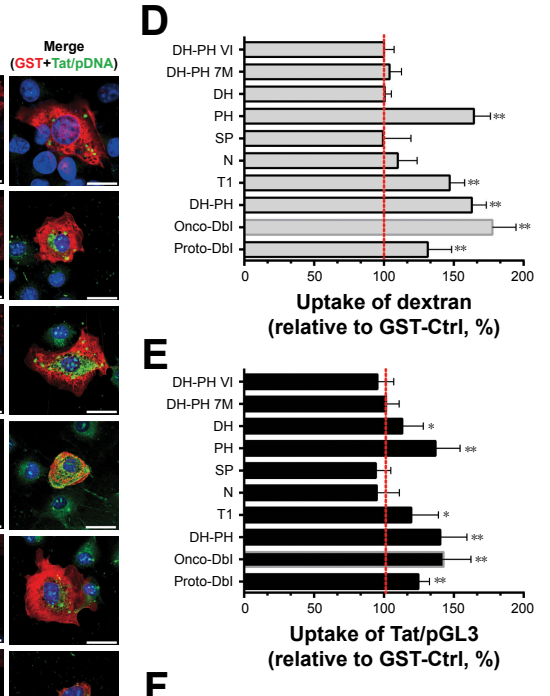

$\mathbf{F}$
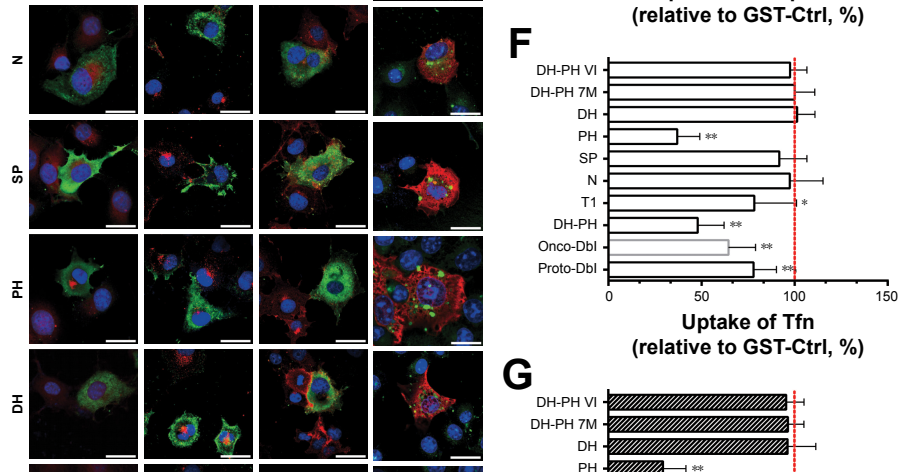

G

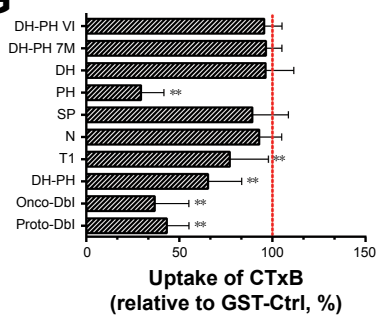

Figure I PH domain of Dbl determines the promotion of Tat/pDNA macropinocytosis.

Notes: (A) Schematic representation of Dbl and its limited-domain constructs. See details in the "Materials and methods" section. (B) Cos-7 cells were transfected with Dbl constructs as listed above. Forty-eight hours post-transfection, the cell lysates were analyzed by Western blotting with mouse anti-GST Ab as described in the "Materials and methods" section. (C) Cos-7 cells were transfected with Dbl constructs as listed in (A). Cells were quickly rinsed with PBS/0.5 M NaCl, growth continued for 48 hours, and then Tfn-AF647, CTxB-AF647, dextran-AF647, and Tat/PGL3-YOYO-I complex were added as described (Table S2; "Materials and methods") at $37^{\circ} \mathrm{C}$. Cell uptake was determined by confocal imaging analysis, and representative images are shown in (C). Scale bar: $20 \mu \mathrm{m}$. (H-K) Scatterplots depict the uptake variability of indicated transfections in single-cell populations. Uptake levels in GST-fusion expressed cells (labeled with mouse anti-GST Ab following by anti-mouse Ab-AF488 or by anti-mouse Ab-AF647) were quantified as described in the "Materials and methods" section. The uptake of pEBG-transfected cells (GST-Ctrl) was set at I00\% for comparison, significant differences compared with proto-Dbl transfected cells are shown, and $\geq 60$ cells were counted for each transfection. (D-G) The uptake levels of Dbl construct-transfected cell populations were analyzed and quantified by fluorescence activated cell sorting. 10,000 cells were counted per transfection in each experiment. Statistically significant differences compared with GST-Ctrl are shown. $* P<0.05$, $* * P<0.0$ I.

labeled ligands at $37^{\circ} \mathrm{C}$, rinsed twice with $\mathrm{PBS} / 0.5 \mathrm{M} \mathrm{NaCl}$, and immediately image-acquired using an Olympus FV 1000 confocal microscope. Software-based quantification analysis of intracellular fluorescent particles was performed as previously described. ${ }^{25,26}$ Polymerized actin and GST-fused protein expressed cells were stained by Rhodamine-phalloidin $(100 \mathrm{nM})$ and the anti-GST antibody (1:200) plus anti-mouse Ab-AF647 (1:1,000), respectively.

\section{Uptake measurement by flow cytometry analysis}

The binding and uptake measurement were performed as previously described. ${ }^{25,26}$ Trypan Blue was added to quench cell-surface-associated fluorescence before cells were harvested for immediate fluorescence activated cell sorting analysis. To compare the uptake in cultures with drug inhibition, medium conditions were achieved in cells via incubation with Rho GTPase inhibitors $(50 \mu \mathrm{M})$ or endocytosis inhibitors (Table S2). Stock solutions of these drugs were made in dimethyl sulfoxide (DMSO), diluted in incomplete medium (w/o FBS), and the final concentration of DMSO was $<0.4 \%$.

\section{Rho GTPase activation assay}

The $\mathrm{Cdc} 42 / \mathrm{RhoA} / \mathrm{Rac} 1$ activation level was checked as previously described. ${ }^{26}$ Serum-starved cells were lysated, and the pre-cleared cell lysate was incubated using 
GST-PAK-CRIB beads (for Cdc42/Rac activation) or GSTRhotekin-RBD beads (for RhoA activation). After subjecting the collected pellets to SDS-PAGE, the GTP-bound Cdc42, RhoA, and Rac1 in samples were determined by Western blotting.

\section{Western blotting}

The protein concentration of the samples was measured using the BCA assay, resolved by SDS-PAGE, and blotted onto PVDF membranes with Abs diluted as follows: anti-Dbl Ab (1:200), anti-GST Ab (1:500), anti-Cdc42 Ab (1:250), antiRhoA Ab (1:250), anti-Rac1 Ab (1:200), and anti-B-actin $\mathrm{Ab}(1: 2,000)$.

\section{Statistical analyses}

All data were obtained from at least three independent experiments and are presented as the mean \pm SD. For two-sample comparisons against the control, the unpaired Student's $t$-test was used unless otherwise noted. One-way analysis of variance with Dunnett's multiple comparison was used to evaluate the statistical significance of at least three groups of samples. Graphs were created using GraphPad Prism five software $(* P<0.05, * * P<0.01)$.

\section{Results}

Noncovalent compact pDNA with HIV-Tat into nanosize complexes pDNA binding is a prerequisite for CPPbased gene delivery. The gel retardation assay showed that Tat peptides were able to completely bind either with
pGL3-YOYO-1 or with the pGL3 plasmid at N/P ratios no less than 5 (Figure $2 \mathrm{~A}$ and $\mathrm{B}$ ). This indicates that Tat peptides are effective in pDNA binding and that the binding ability is not susceptible to YOYO-1 dye labeling. The condensation of pDNA into particulate structures is essential for gene delivery. For this reason, we next examined the capability of Tat peptides to condense pDNA and to form particulate complexes. Laser light scattering results showed that all of the Tat/pGL3-YOYO-1 complexes had an average diameter $>1 \mu \mathrm{m}$ when the $\mathrm{N} / \mathrm{P}$ ratios were no more than 5 , which decreased to $\sim 600 \mathrm{~nm}$ in PBS buffer $(150 \mathrm{mM} \mathrm{NaCl}$, $\mathrm{pH} 7.4)$ or in DMEM (110 mM NaCl, pH 7.4) when the N/P ratios were no less than 10 (Table 1). Similar size changes of the Tat/pGL3 complexes were seen when the N/P ratios were $\geq 5$. Both Tat/pGL3-YOYO-1 and Tat/pGL3 complexes had a negative $\zeta$ potential of approximately $-17 \mathrm{mV}$ to $-41 \mathrm{mV}$ at an N/P ratio of 1 , which changed to a positive $\zeta$ potential more than $+1 \mathrm{mV}$ and markedly increased with the enhanced amount of peptides (Table 1). The gradually increased positive $\zeta$ potential and the particle size remained stable when $\mathrm{N} / \mathrm{P}$ ratios were $>5$, indicating the formation of compact complexes.

The degree of pDNA compaction in the complex was further determined by the DNase I protection assay. Either pGL3 or pGL3-YOYO-1 in the complexes prepared with an $\mathrm{N} / \mathrm{P}$ ratio of 1 was only partially protected from DNase I digestion. The total resistances to the action of DNase I were observed in the complexes obtained with N/P ratios $>5$. When high $\mathrm{N} / \mathrm{P}$ ratios were used, complexes formed in both $\mathrm{PBS}$ or in DMEM provided extensive protection
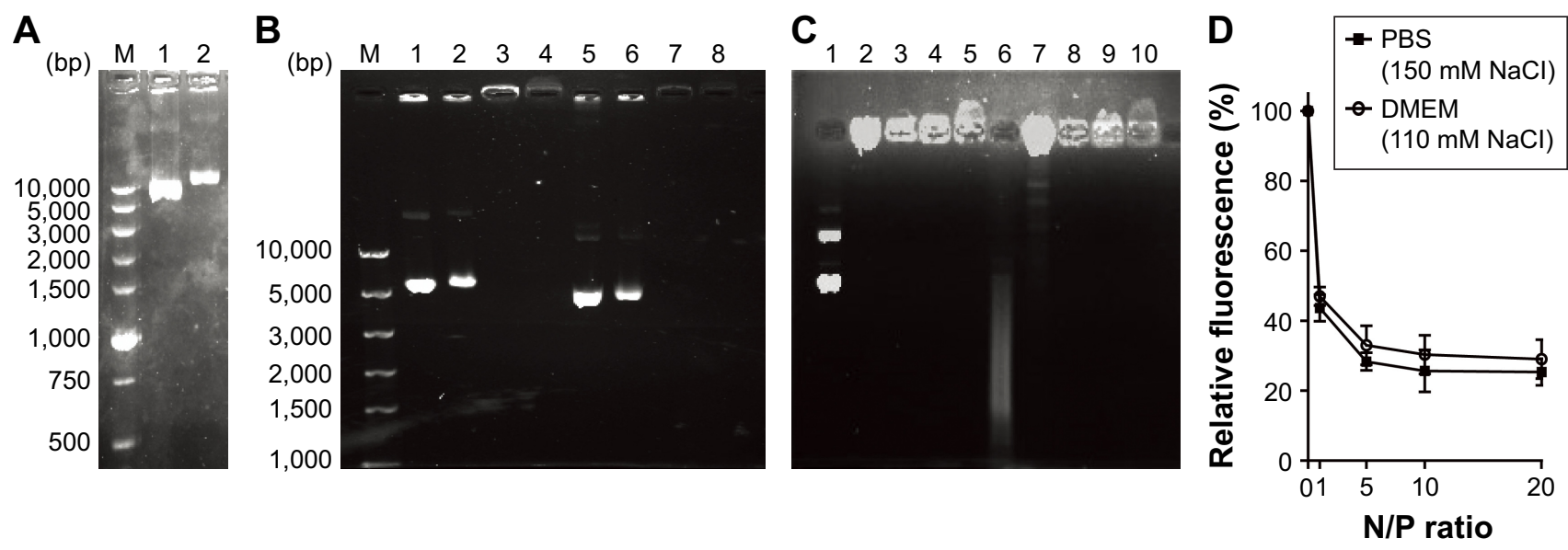

Figure 2 Compact pDNA with HIV-Tat into nano-size Tat/pDNA complexes.

Notes: (A) Agarose electrophoretic mobility assay. The ratio for YOYO-I-labeled pDNA was I:5 (D:BP). M: DNA ladder; I: pGL3; 2: pGL3-YOYO-I. (B) Gel retardation assay. M: DNA ladder; lanes I-4: Tat/pGL3-YOYO-I complexes; lanes 5-8: Tat/PGL3 complexes; in both groups, the N/P ratios between Tat and pGL3 were 0, I, 5, 20, respectively. (C) DNase I protection assay. Lanes I-5: Tat/PGL3-YOYO-I complexes (N/P=0, I, 5, I0, 20, respectively) without DNase I treatment. Lanes 6-I0: corresponding Tat/PGL3-YOYO-I complexes with DNase I treatment. (D) Fluorescent quenching assay. The relative fluorescent intensity ratio depending on the charge ratio of Tat/PGL3YOYO-I complexes is plotted with respect to the fluorescence of PGL3-YOYO-I before condensation. YOYO-I-labeled pGL3 was completed with the Tat peptide with increasing charge ratios. 
Table I Particle size $(\mathrm{nm})$ and $\zeta$ potential $(\mathrm{mV})$ of Tat/pDNA complexes

\begin{tabular}{|c|c|c|c|c|}
\hline N/P ratio & $\mathbf{I}$ & 5 & 10 & 20 \\
\hline \multicolumn{5}{|l|}{ Tat/pGL3-YOYO-I } \\
\hline PBS (I50 mM NaCl) & $3,930 \pm 85(-29.2 \pm 3.1)$ & $\mathrm{I}, 35 \mathrm{I} \pm 36(1.9 \pm 0.9)$ & $599 \pm 49(9.9 \pm 2.1)$ & $606 \pm 17(13.9 \pm 6.2)$ \\
\hline DMEM (I I 0 mM NaCl) & $4,742 \pm 112(-17.9 \pm 2.2)$ & $2,222 \pm 69(1.1 \pm 0.6)$ & $595 \pm 81(8.4 \pm 0.8)$ & $619 \pm 15(10.9 \pm 5.1)$ \\
\hline \multicolumn{5}{|l|}{ Tat/pGL3 } \\
\hline PBS (I50 mM NaCl) & $I,|55 \pm 4|(-4 \mid .2 \pm 9.2)$ & $761 \pm 33(4.2 \pm 2.0)$ & $5|6 \pm 2|(1 \mid .2 \pm 4.6)$ & $514 \pm 30(18.2 \pm 7.5)$ \\
\hline DMEM (I IO mM NaCl) & $1,040 \pm 63(-40.2 \pm 8.3)$ & $606 \pm 27(4.0 \pm 1.2)$ & $332 \pm 28(10.2 \pm 3.1)$ & $338 \pm 15(14.2 \pm 6.7)$ \\
\hline
\end{tabular}

Notes: Tat/pGL3 and Tat/pGL3-YOYO-I complexes were prepared in different buffers at the indicated charge ratios. The particle size (up value) and $\zeta$ potential (down value) were measured.

from nucleases (Figure 2C). Moreover, the compaction extent of complexes was also analyzed by the fluorescent quenching assay. YOYO-1 fluorescent quenching was strongly increased at low charge ratios, reached a plateau at an N/P ratio of 5, and only slightly increased at higher ratios for further pGL3-YOYO-1 condensation (Figure 2D). Obviously, the low remaining fluorescent level $(<40 \%)$ of YOYO-1 after Tat competition-induced fluorescent quenching further indicated that pDNA was tightly packed into nano-size complexes at the physiological level of ionic strength.

\section{Medium-sized tat/pDNA nano-complexes mainly use macropinocytosis for uptake}

As debate still exists on the delivery of CPP-based nucleic acid drugs, ${ }^{27,28}$ we next examined the uptake mechanism of Tat/pDNA complexes. For each of the N/P ratios, transfections performed both at $4{ }^{\circ} \mathrm{C}$ and in the presence of metabolic inhibitors led to a strong reduction of Tat/pGL3 transgene expression in Cos-7 cells (Figure 3A and B). In contrast, transfection efficiencies in the presence of chloroquine, which reduces endolysosomal entrapment and degradation of Tat/pGL3 complexes, were enhanced 2- to 7-fold (Figure 3C). Similar trends were observed for the uptake of Tat/pGL3-YOYO-1 complexes when checked by confocal imaging analysis. These data indicate that the uptake of Tat/pDNA complexes was apparently through endocytosis and not mediated by direct membrane penetration.

Seeing that several uptake routes (eg, CME, CvME, and macropinocytosis) are possibly involved in endocytosis, we next determined the endocytosis pathway required for Tat/pDNA complexes by using various markers and inhibitors (Table S2). Confocal imaging analysis showed that Tat/pGL3-YOYO-1 complexes with an N/P ratio of 10 were not co-localized with the CME marker Tfn-AF647 and lipid raft marker CTxB-AF647 (Figure S1). This contrasts with the majority of the complexes that were co-localized with the macropinocytosis marker dextranAF647 (Figure 3D). Similar co-localization patterns were observed for Tat/pGL3-YOYO-1 complexes with an N/P ratio of 20. For Tat/pGL3-YOYO-1 complexes with lower $\mathrm{N} / \mathrm{P}$ ratios, the total number of intracellular pDNA spots was markedly reduced, and no pDNA spots were found in cells transfected with pGL3-YOYO-1 vectors. The contribution of macropinocytosis to the uptake of medium-sized $(\sim 600 \mathrm{~nm})$ Tat/pDNA complexes under non-stimulating condition was further verified. Cos-7 cells pretreated with the CME inhibitor CPZ did not inhibit Tat/pGL3 (N/P=5) transgene expression. The CvME inhibitor filipin did not significantly inhibit this transgene expression. The cholesterol-depleting reagent $\mathrm{MBCD}$, which destroys the caveolae-required lipid raft structure, however, slightly enhanced this transgene expression. When Cos-7 cells were pretreated either with the macropinocytosis inhibitor EIPA or with $\mathrm{CyD}$, the transgene expression of the Tat/pGL3 complexes (N/P=5) was significantly inhibited (Figure 3E). In addition, similar uptake changes of Tat/pGL3-YOYO-1 ( $/ \mathrm{P}=10,20)$ complexes were observed when using these inhibitors in the flow cytometry assay (Figure 3F). These results suggested that macropinocytosis is the dominant uptake pathway for medium-sized $(\sim 600 \mathrm{~nm})$ Tat/pDNA complexes.

\section{Rho GTPases are involved in cell-type- specific macropinocytosis of tat/pDNA complexes}

To further investigate the contribution of macropinocytosis to the uptake of medium-sized ( $\sim 600 \mathrm{~nm})$ Tat/pDNA complexes, more available cell lines (tumor and non-tumor) in our laboratory were included in the luciferase gene expression assay. Different levels of luciferase expression were observed after Tat/pGL3 (N/P=5) complex transfections (Figure 4A), which sharply contrasted with the non-significant expression changes in $\mathrm{N} / \mathrm{P}=10$ or $\mathrm{N} / \mathrm{P}=20$ groups (Figure 4B). In view of the co-localization of Tat/ pGL3-YOYO-1 complexes with dextran-AF647 (Figure 3D), 
A

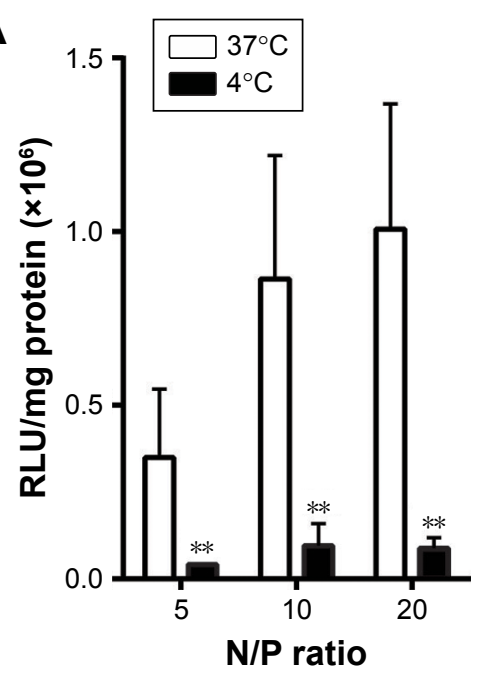

\section{B}

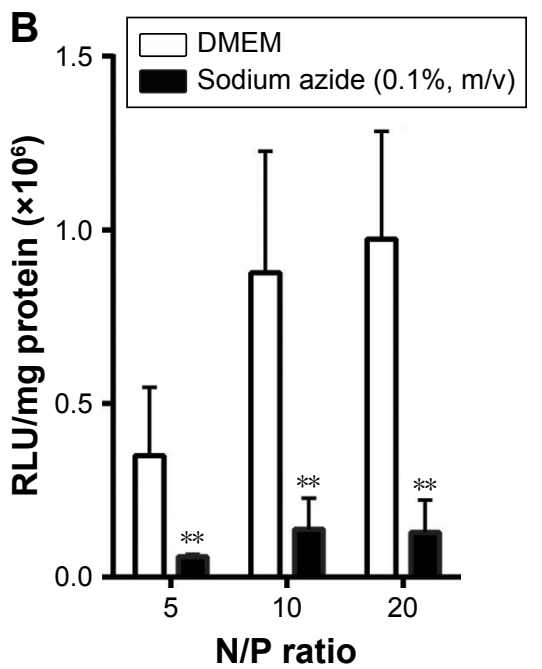

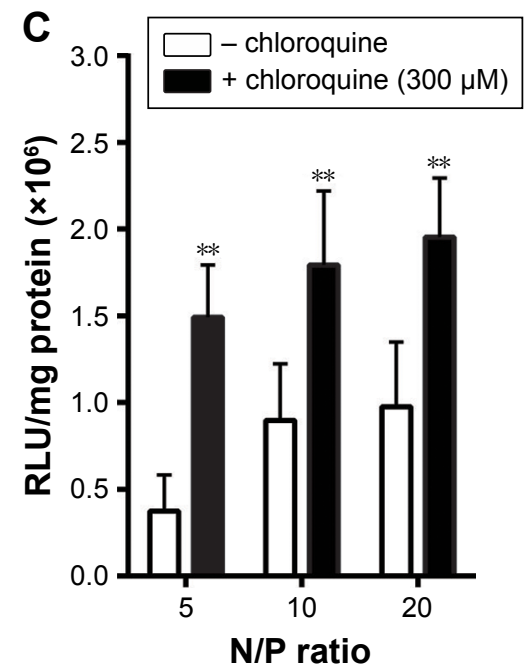

D
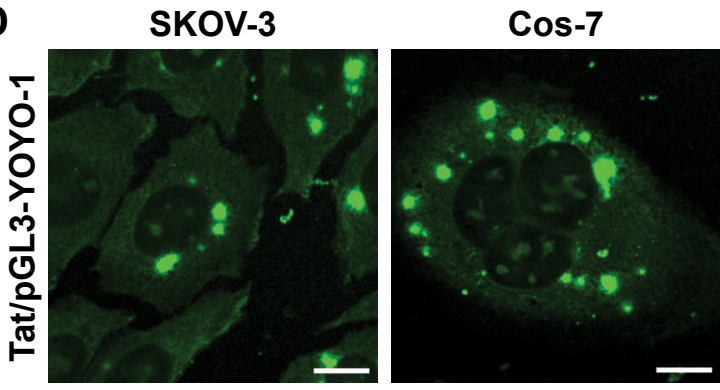

E
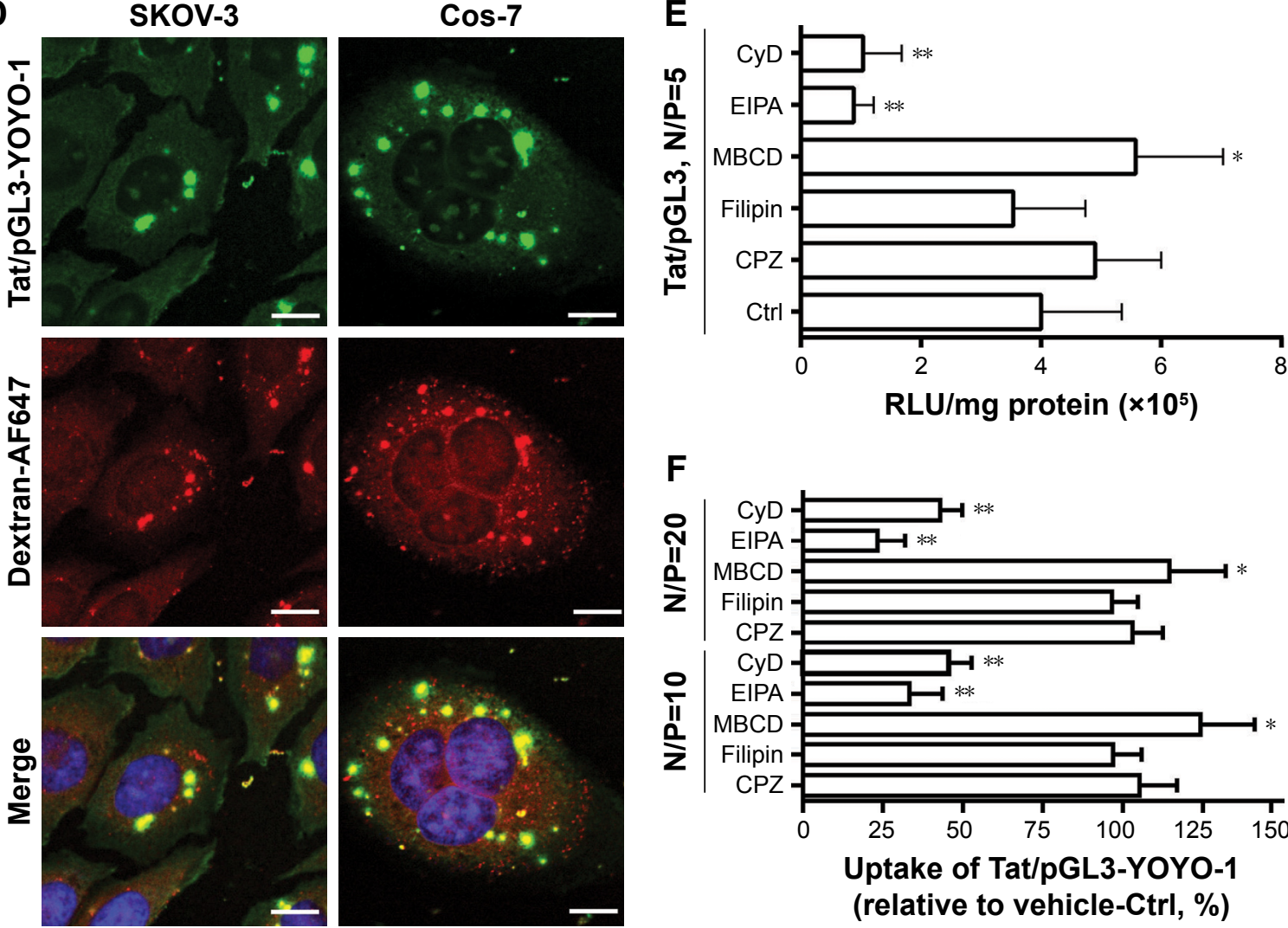

$\mathbf{F}$
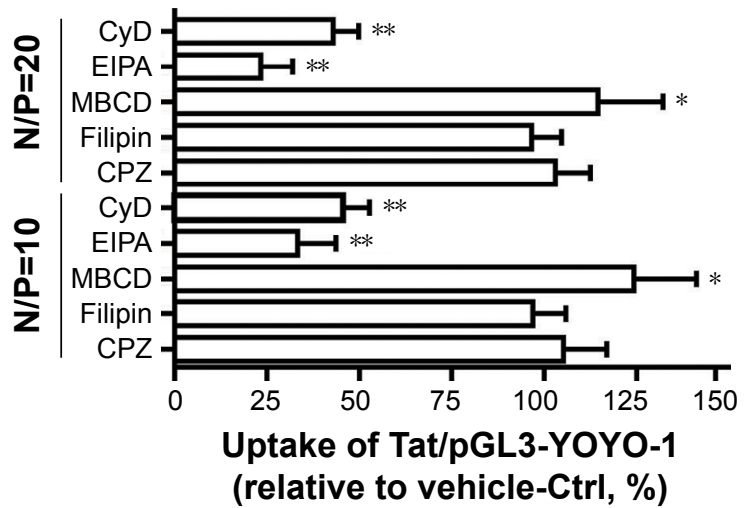

Figure 3 Nano-size Tat/pDNA complexes mainly use macropinocytosis for uptake.

Notes: Luciferase activity was determined in COS-7 cells transfected with different N/P ratios of Tat/pGL3 complexes under the following conditions: (A) at different temperatures, $(\mathbf{B})$ in the absence or presence of sodium azide $(0.1 \%, \mathrm{~m} / \mathrm{V})$, (C) with/without chloroquine $(300 \mu \mathrm{M})$. RLU/l0 S/mg protein, relative light units (RLU) of expressed as luciferase per milligram protein in 10 seconds in detection mode. (D) Confocal imaging analysis of intracellular localization of Tat/pGL3-YOYO-I complexes. COS-7 and SKOV3 cells were incubated with Tat/PGL3-YOYO-I complexes (N/P=10, green emission signal) and dextran-AF647 (red emission signal) simultaneously for I hour. Cell nuclei were stained with Hoest33/2 (blue emission signal). (E) Luciferase activity was determined in Tat/pGL3 (N/P=5) transfected COS-7 cells, which were treated with endocytosis inhibitors (Table S2). Transfection rates in inhibitor-treated cells significantly differed from that of DMSO $(<0.4 \%$, final concentration) treated cells (Ctrl) are indicated. ${ }^{*} \mathrm{P}<0.05$, ${ }^{*} * \mathrm{P}<0.0 \mathrm{I}$. (F) The uptake levels of Tat/pGL3-YOYO-I complexes (N/P=I0, 20) in Cos-7 cells, pretreated with endocytosis inhibitors, were quantified and analyzed by fluorescence activated cell sorting. About 10,000 cells were counted per transfection in each experiment. Statistically significant differences compared with dimethyl sulfoxide (DMSO) $(<0.4 \%$, final concentration) treated cells (Ctrl) are shown, $* P<0.05$, $* * P<0.01$.

this contrasted transfection expression suggests that there might be cell-type-specific macropinocytosis variations. In fact, when checking the uptakes of endocytosis markers and Tat/pGL3-YOYO-1 complexes $(\mathrm{N} / \mathrm{P}=10)$ by flow cytometry, there were less different internalizations of Tfn-AF647 and CTxB-AF647 among these cells, but the highest uptake levels of dextran-AF647 and Tat/pGL3-YOYO-1 complexes in SKOV3 cells were observed (Figure 4C). These two 
A

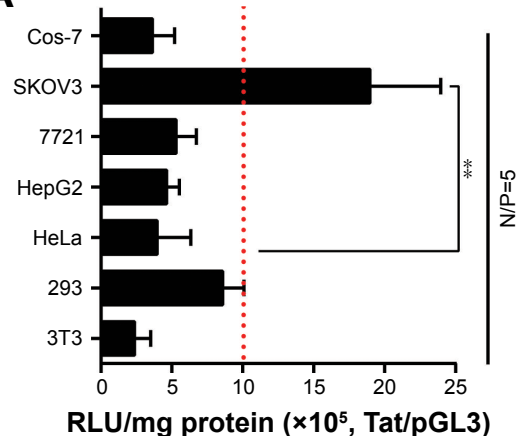

C

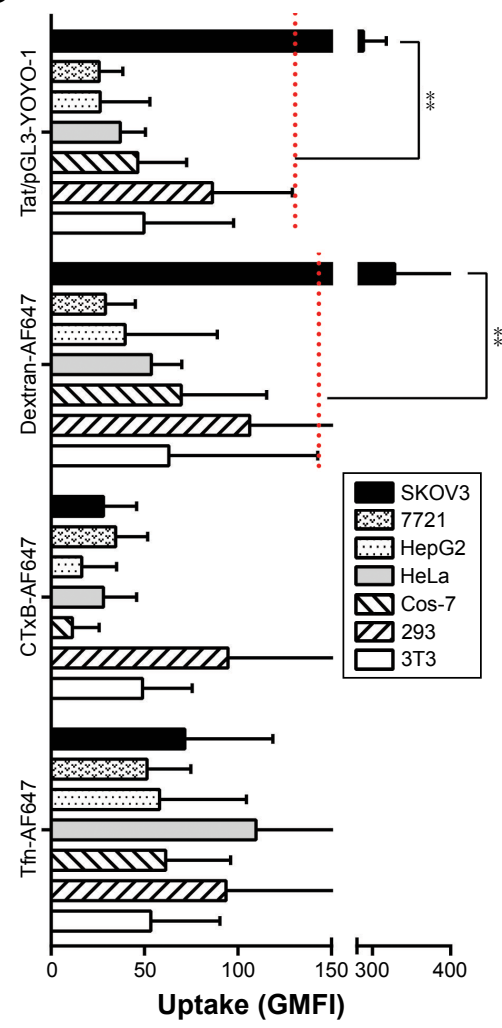

B

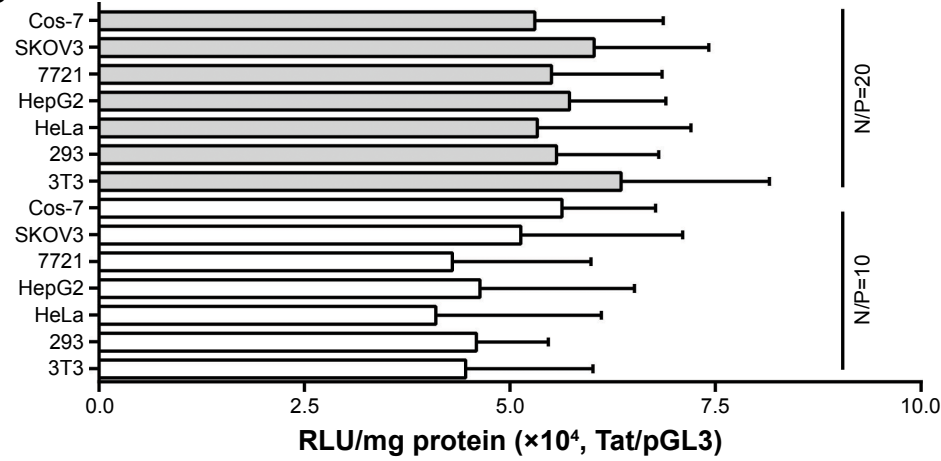

D
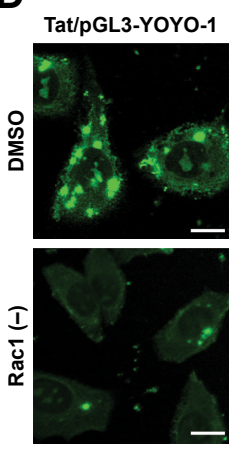

Dextran-AF647

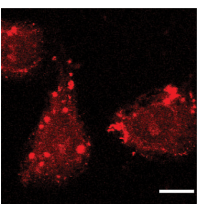

E
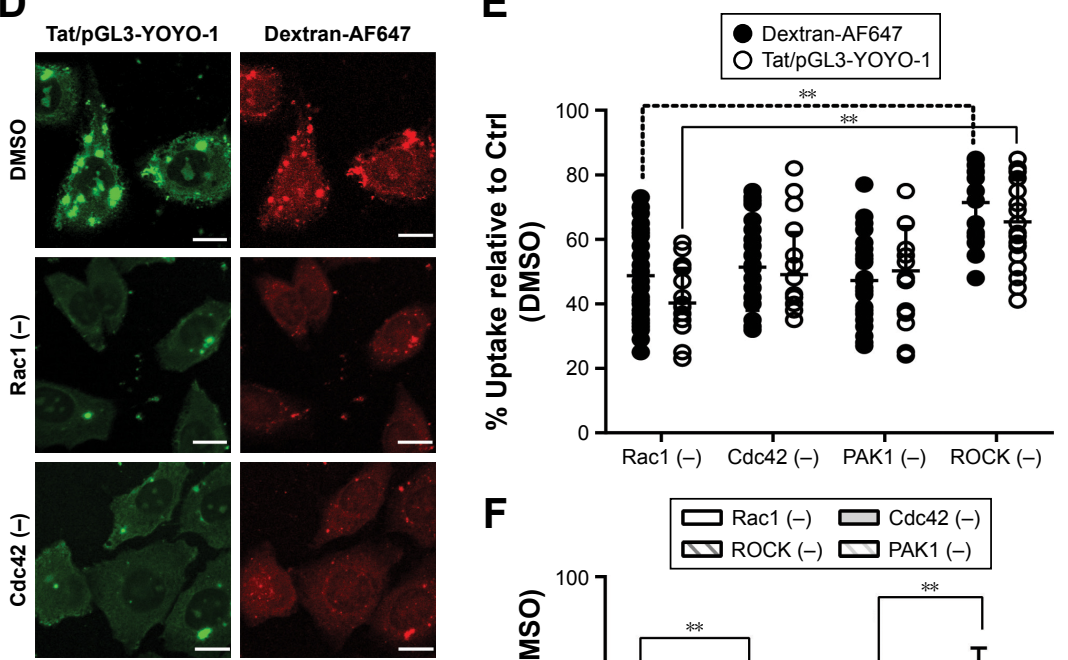

$\mathbf{F}$
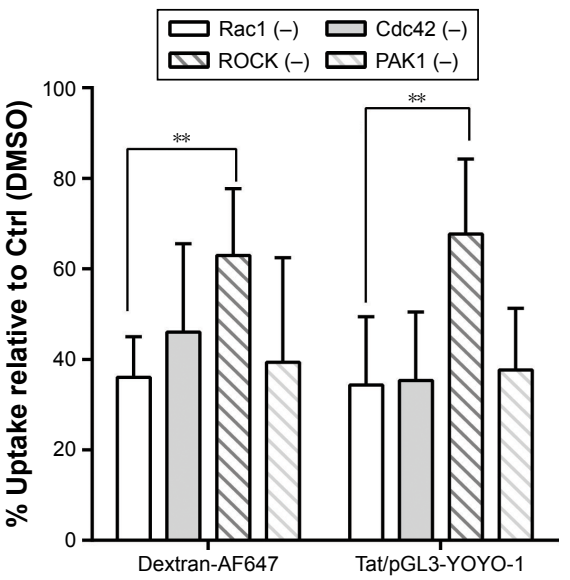

Figure 4 Rho GTPases are involved in the macropinocytosis of Tat/pDNA complexes.

Notes: Luciferase activity was determined in tumor and non-tumor cell lines transfected with Tat/pGL3 complexes at N/P ratios of 5 (A), 10 , and 20 (B). The uptakes of CME marker (Tfn-AF647), lipid raft marker (CTxB-AF647), macropinocytosis marker (dextran-AF647), and Tat/pGL3-YOYO-I complexes (N/P=I0, 20) were quantified by fluorescence activated cell sorting and compared among tumor and non-tumor cell lines (C). About 10,000 cells were counted per sample in each experiment. Confocal imaging analysis of the uptakes of dextran-AF647 and Tat/PGL3-YOYO-I complexes (N/P=10) in the presence of different Rho GTPase inhibitors " $(-)$ " as described in the "Materials and methods" section. Representative images are shown in (D). Scatterplots depict the uptake changes of indicated ligands in single cell populations (E). About $\geq 60$ cells were counted for each treatment in each experiment. The uptake changes of Tat/pGL3-YOYO-I complexes (N/P=10) in SKOV3 cells, treated with different Rho GTPase inhibitors ( $<0.4 \%$ dimethyl sulfoxide [DMSO] as Ctrl), were quantified and analyzed by fluorescence activated cell sorting $(\mathbf{F})$. About I0,000 cells were counted per sample in each experiment. $* * P<0.01$.

aspects together revealed that macropinocytosis of mediumsized ( $\sim 600 \mathrm{~nm})$ Tat/pDNA complexes in SKOV3 cells is uniquely high.

Macropinocytosis is generally regulated by several signaling pathways, most of which involve Rho GTPases. ${ }^{29}$ It is unclear whether the medium-sized ( $\sim 00 \mathrm{~nm})$ Tat/pDNA complex was internalized via the same signaling pathway.
To address this issue, SKOV3 cells were preincubated with Rho GTPase inhibitors for 1 hour before Tat/pGL3-YOYO-1 complexes $(\mathrm{N} / \mathrm{P}=10)$ were added for uptake. Confocal imaging showed that Rac1 and Cdc42 inhibitors strongly decreased the uptake of Tat/pGL3-YOYO-1, and the ROCK inhibitor partially blocked the uptake of Tat/pGL3-YOYO-1 (Figure 4D and E). PAK1 is a downstream signal mainly 
activated by $\mathrm{Cdc} 42$ and Rac. As expected, the PAK1 inhibitor blocked Tat/pGL3-YOYO-1 uptake just as the Cdc42 and Rac1 inhibitors did (Figure 4D and E). However, a relatively less impaired uptake of Tat/pGL3-YOYO-1 was observed when cells were treated with the ROCK inhibitor (RhoA effector). Intracellular fluorescent particles were also counted by flow cytometry, and the uptake levels were significantly lower in the cells incubated using Tat/pGL3-YOYO-1 with inhibitors than in those incubated by Tat/pGL3P-YOYO-1 alone (Figure 4F). The expression of inhibition of Tat/ pGL3-YOYO-1 complexes (N/P=5) was similarly observed in SKOV3 cells treated with Rho GTPase inhibitors (Figure S2). Apparently, Rho GTPase signaling from Rac1, $\mathrm{Cdc} 42$, and RhoA are all involved in the macropinocytosis of Tat/pDNA complexes.

\section{Dbl increases the macropinocytosis of tat/ pDNA complexes in ovarian cancer cells}

Despite Rho GTPases being involved in Tat/pDNA macropinocytosis, it remains unclear why the macropinocytosis level is distinctly high only in SKOV3 cells. As Rho GTPases are always activated by upstream factors mainly through Dbl family members, ${ }^{30,31}$ we then investigated whether Dbl, the representative prototype of this oncogenic family, plays a unique role in cell-specific signaling that may differentially regulate macropinocytosis activity. Western blot of these cell lines showed that proto-Dbl was expressed at the highest level in SKOV3 cells but less expressed in other cells and not expressed in Cos-7 cells. Remarkably, the onco-form of Dbl (onco-Dbl) only appeared in SKOV3 cells (Figure 5A). Interestingly, when the internalization of endocytosis markers was analyzed, both confocal imaging and flow cytometry analysis showed that the uptake of dextran-AF647 was most markedly high in SKOV3 cells (Figures $4 \mathrm{C}$ and $5 \mathrm{~B}$ and $\mathrm{C}$ ). It seems that high proto-Dbl expression and unique onco-Dbl presentation are positively correlated with the dominating macropinocytosis activity in SKOV3 cells.

To directly determine the role of $\mathrm{Dbl}$ on macropinocytosis regulation, Dbl-siRNA-transfected SKOV3 cells were overexpressed with proto-Dbl and onco-Dbl constructs (Figure 5D). We found that Dbl knockdown not only significantly decreased the uptake of dextran-RITC (average decrements $45 \%$ and $40 \%$ were obtained in FCM and confocal analyses, respectively) but also decreased the uptake of Tat/pGL3-YOYO-1 complexes (average decrements 45\% and $40 \%$ were obtained in FCM and confocal analyses, respectively). Notably, the knockdown-decreased uptakes of dextran-RITC and Tat/pGL3-YOYO-1 were only partially reversed by proto-Dbl overexpression but completely reversed by onco-Dbl overexpression (Figure $5 \mathrm{E}-\mathrm{H}$ ). No significant uptake changes of Tfn-AF647 and CTxB-AF647 were observed after Dbl gene modulations (Figure S3A and B). In addition, the rescued macropinocytosis of Tat/pGL3YOYO-1 by onco-Dbl overexpression mirrored the increased transgene expression of Tat/pGL3 complexes $(\mathrm{N} / \mathrm{P}=5)$ in $\mathrm{Dbl}$ knockdown SKOV3 cells (Figure 5I). These results strongly support that onco-Dbl, but not proto-Dbl, promotes the macropinocytosis of Tat/pDNA complexes in SKOV3 cells.

\section{Dbl hyperstimulated macropinocytosis via $\mathrm{PH}$ domain mediated by Racl activation}

$\mathrm{Dbl}$ is the putative GEF (guanine nucleotide exchange factor) for Rho GTPases containing tandem sec14-like, spectrin-like (SP), Dbl homology (DH), and pleckstrin homology $(\mathrm{PH})$ domains..$^{23,25,30,31}$ To examine which domain of Dbl determines the macropinocytosis promotion, Cos-7 cells were transfected with full-length Dbl or its truncated domain plasmids (Figure 1A and B), and the uptakes of Tat/pGL3-YOYO-1 and endocytosis markers were compared. Compared with onco-Dbl, PH, or DH-PH expression alone similarly significantly increased the macropinocytosis of Tat/pGL3-YOYO-1 and dextran-AF647 (Figure 1C-E, H, and I). DH or T1 expression alone resulted in only a small macropinocytosis increase for Tat/pGL3-YOYO-1 (Figure 1C, E, and I) but had different effects on dextran-AF647 uptake (Figure 1C, D, and $\mathrm{H}$ ). No uptake changes of these drugs were observed among the remaining transfections. In contrast, the uptakes of CTxb-AF647 and Tfn-AF647 were both inhibited by $\mathrm{PH}$ or DH-PH expression (Figure 1C, F, G, J, and K). Thus, the role of onco-Dbl in the promotion of Tat/pDNA macropinocytosis is likely determined by the $\mathrm{PH}$ domain, but not by the DH domain.

Rho GTPases are the master regulators for macropinocytosis, and their activity is stimulated by upstream growth factors mediated by $\mathrm{Dbl}$ family members. ${ }^{25,30}$ To fully determine the promotion role of onco- $\mathrm{Dbl}$ in macropinocytosis, we further examined the effects of Dbl expression on Rho GTPase activation in Cos-7 cells. Onco-Dbl expression induced nearly five times the increment in Rac1 activation, and nearly three times the increment in $\mathrm{Cdc} 42$ and RhoA activation. However, proto-Dbl expression did not significantly induce RhoA activation but induced a small increment in Cdc42 and Rac1 activation (Figure 6A and B). Compared with onco-Dbl transfection, expression of $\mathrm{DH}-\mathrm{PH}$ or PH domain similarly increased Rac1 activation 
A

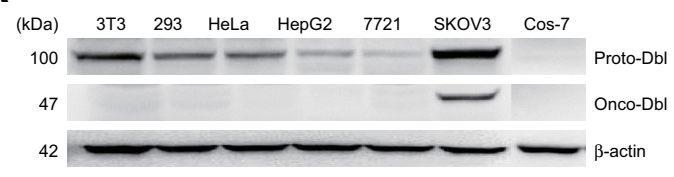

D

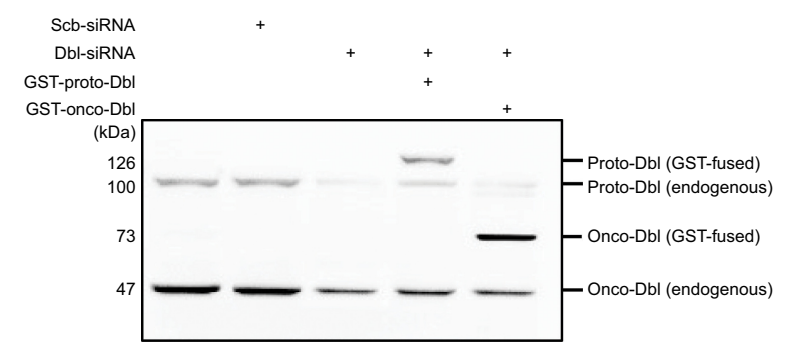

B
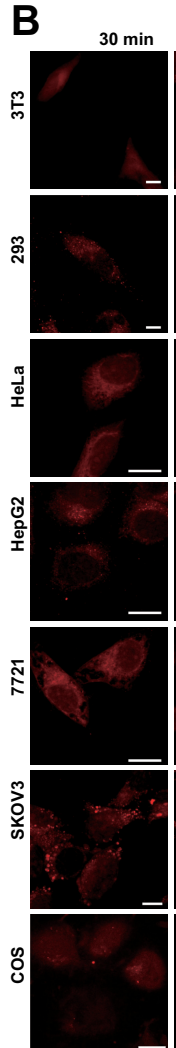

C

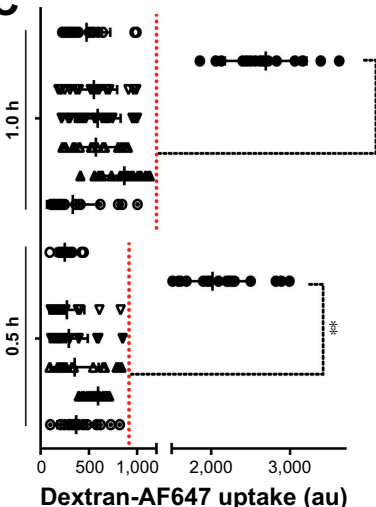

O COS-7 SKOV3 $\nabla 772$

D Hepg2 $\triangle$ HeLa $\Delta 293$ ๑ 3 T3

\section{I}

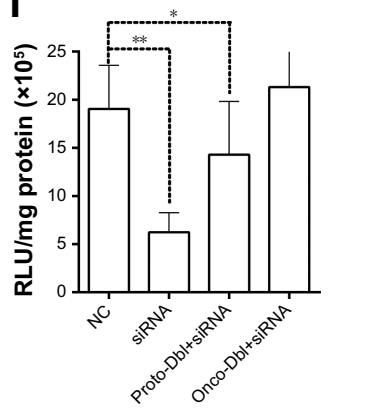

E

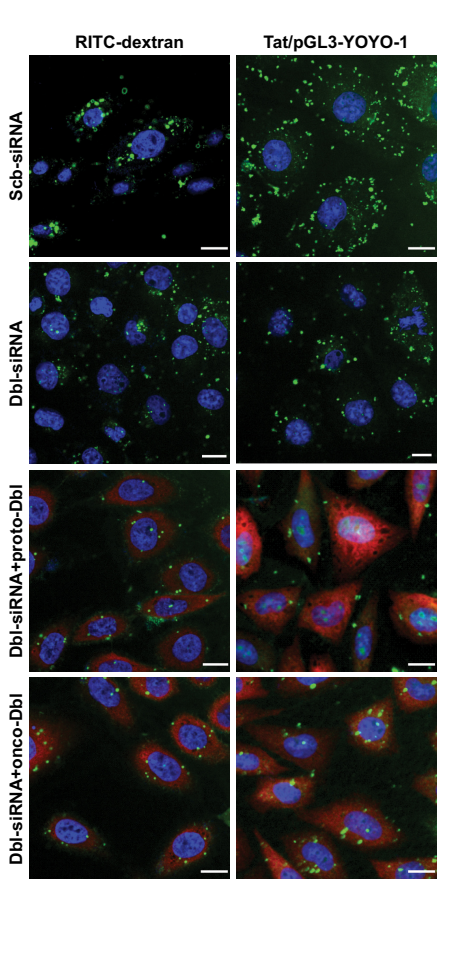

F

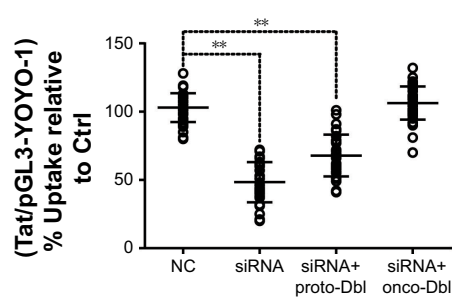

G

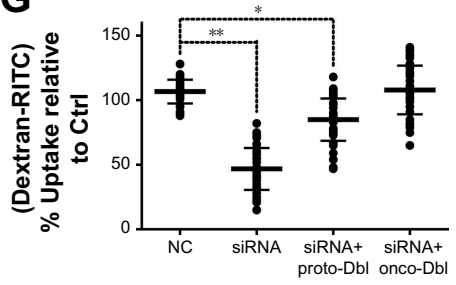

H

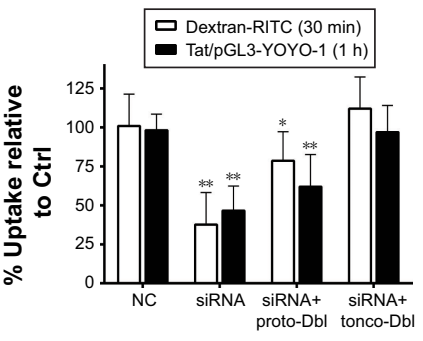

Figure 5 Onco-Dbl promotes the macropinocytosis of Tat/pDNA complexes.

Notes: (A) Western blot determined the expression level of proto- and onco-Dbl in available cell lines (tumor and non-tumor). (B) Confocal imaging analyzed the dynamic difference of macropinocytosis among cell lines. Representative images are shown. Scale bar: $20 \mu \mathrm{m}$. (C) Scatterplots depict the uptake changes of dextran-AF647 in single cell populations. $\geq 60$ cells were counted for each cell line. (D) SKOV3 cells were first transfected with the Dbl-targeted siRNA pool (Dbl-siRNA) or scrambled siRNA (scb-siRNA) and then transfected with GST-tagged proto-Dbl or onco-Db plasmids for rescued overexpression. The cell lysates were analyzed by Western blotting with rabbit anti-Dbl Ab as described in the "Materials and methods" section. (E) Confocal imaging analyzed the uptakes of dextran-RITC and Tat/pGL3-YOYO-I complexes in Dbl-siRNA transfected SKOV3 cells rescued by Dbl-GST plasmid overexpression (labeled by anti-GST Ab with anti-mouse Ab-AF647 staining). Representative images are shown. Scale bar: $20 \mu \mathrm{m}$. (F, G) Scatterplots depict the uptake changes of indicated drugs in single cell populations. Uptake levels in scb-siRNA transfected cells, NC, were set as $100 \%$ for comparison. $\geq 60$ cells were counted for each transfection. $(\mathbf{H})$ The uptake levels of dextran-RITC and Tat/pGL3-YOYO-I complexes from transfected populations were analyzed and quantified by fluorescence activated cell sorting. About 10,000 cells were counted per transfection in each experiment. Statistically significant differences compared with scb-siRNA (NC) transfected cells are shown. (I) Luciferase activity was determined in Dbl-siRNA transfected SKOV3 cells rescued by Dbl-GST plasmid overexpression. Luciferase expression level in scb-siRNA (NC) transfected cells was set as $100 \%$ for comparison. $* P<0.05$, $* * P<0.01$.

(330\% and $280 \%$, respectively, on average), but relatively less increased the activation of Cdc42 (only $180 \%$ and $170 \%$, respectively, on average) and RhoA (only $150 \%$ and $130 \%$, respectively, on average). It is noteworthy that $\mathrm{DH}$ domain expression did not significantly induce the activation of Cdc42 and RhoA, but only slightly increased Rac1 activation (Figure 6A and B). In addition, no Rac1, Cdc42, or RhoA activations were detected after transfection with other indicated plasmids. These results showed that expression of
onco-Dbl, particularly the PH domain, highly stimulates Rac1 activation, whereas expressions of proto-Dbl and its other domains only induced limited Rac1 activation.

Rac1 activation has long been known to drive macropinocytosis upregulation by controlling cytoskeleton dynamics. ${ }^{7,22}$ To examine whether macropinocytosis promotion induced by onco-Dbl was because of enhanced actin dynamics, we investigated the effects of $\mathrm{Dbl}$ expression on cytoskeleton organization change. Onco-Dbl and DH-PH 

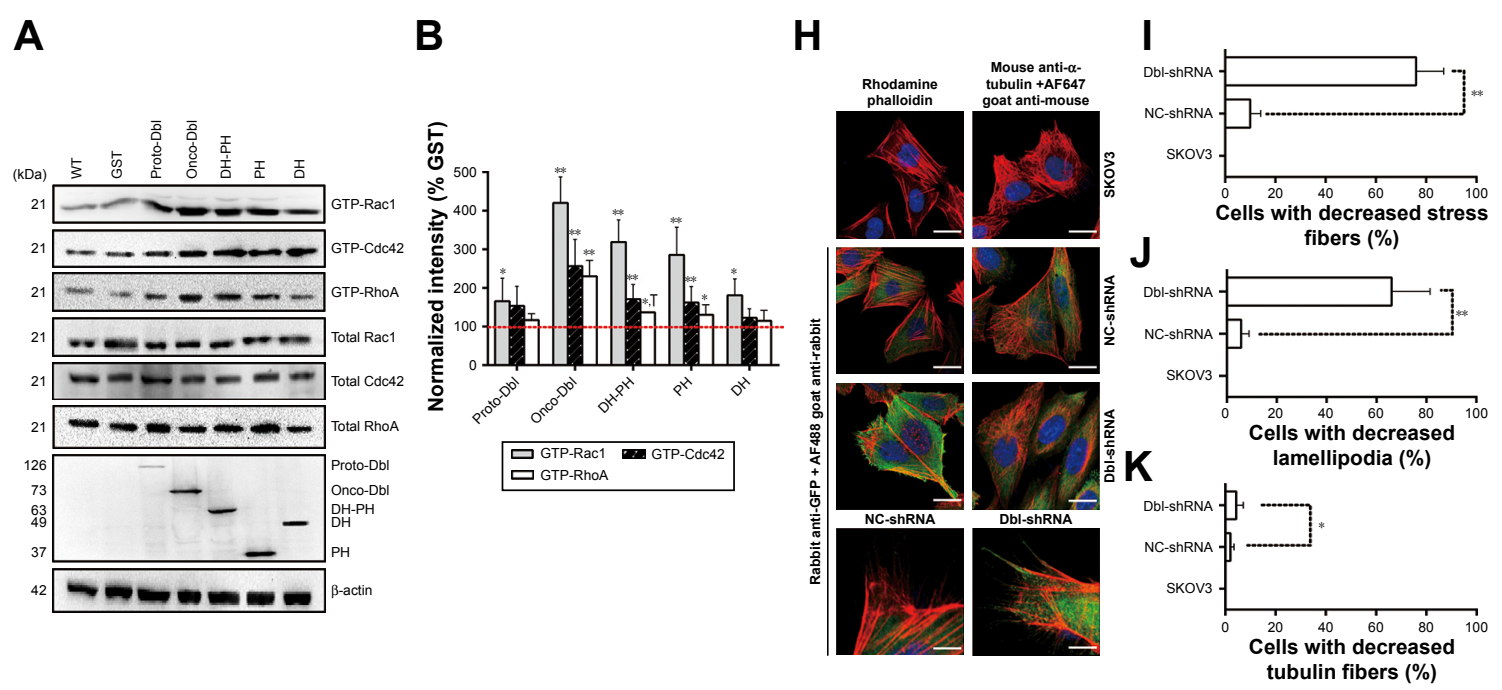
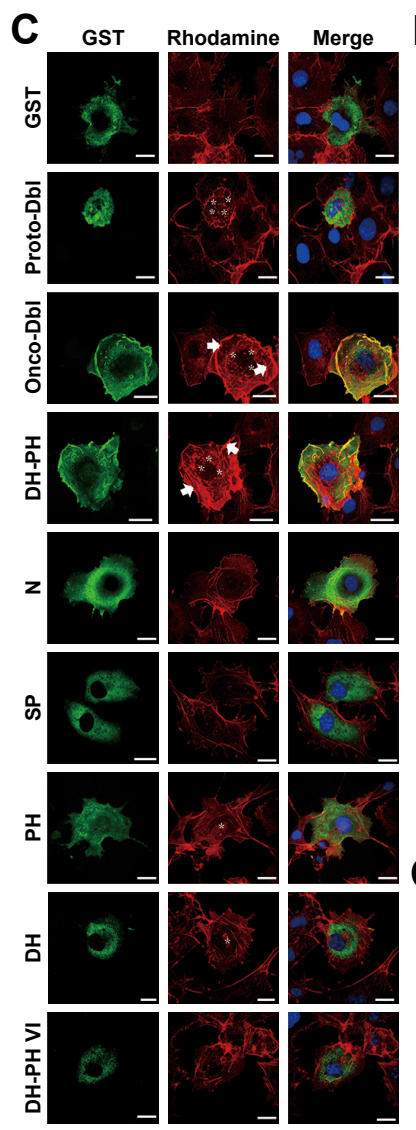

D

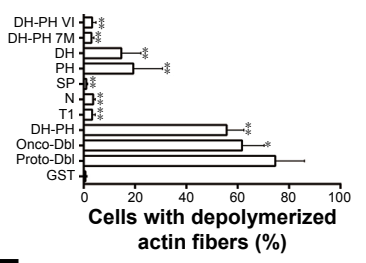

$E$

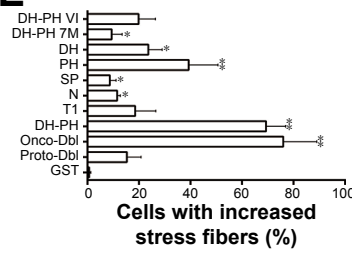

$F_{0}$

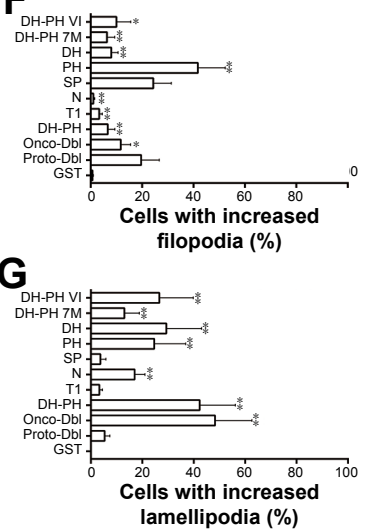

L
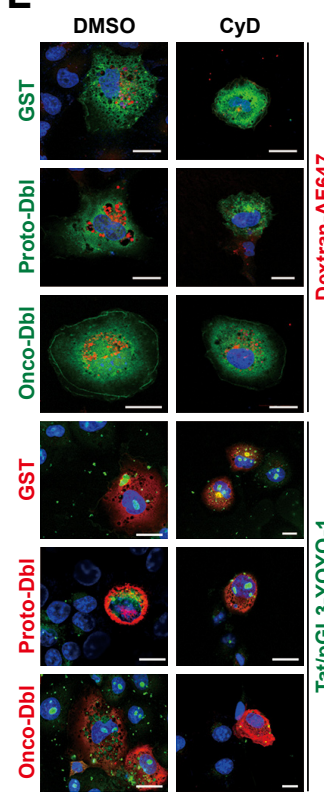

M

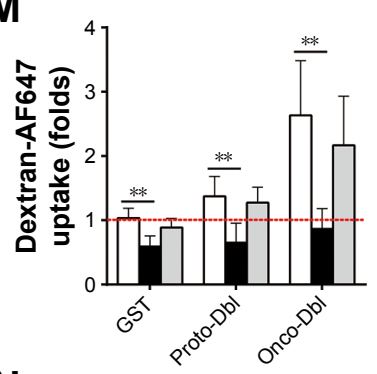

$\mathbf{N}$

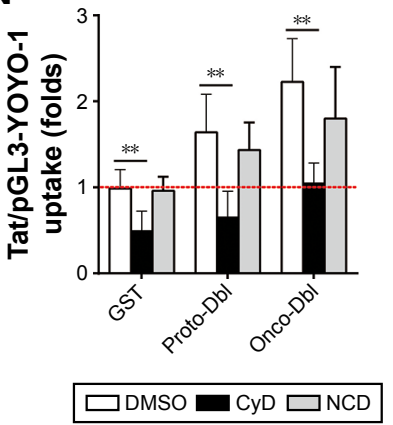

Figure 6 Dbl promotes macropinocytosis via cytoskeleton reorganization mediated by the PH domain and Racl activation.

Notes: (A) Cos-7 cells were transfected with GST-fused Dbl or its limited-domain constructs, serum-starved for 16 hours, and then the fractions of activated Racl, Cdc42, and RhoA were measured via the GST pull down method as described in the "Materials and methods" section. Prime Abs with mouse anti-GST, rabbit anti-Racl, antiCdc42, anti-RhoA, and anti-b-actin were used for Western blotting. Representative blot results from three experiments are shown. (B) Quantitative analysis of the Racl, $\mathrm{Cdc} 42$, and RhoA activation levels were determined via densitometry. Percentage changes were correspondingly corrected with the expression levels of Dbl constructs. Statistically significant differences in relation to the control (pEBG-GST) are shown. (C) Dbl constructs (Figure IA) transfected Cos-7 cells were reseeded in poly-D-lysine pre-coated chamber slides, serum-free starved, and then fixed, permeabilized, and stained with Rhodamine-phalloidin for the detection of polymerized actin. Representative confocal images from three experiments are shown. Scale bar, $20 \mu \mathrm{m}$. (D-G) Morphometric analyses of the extent of filopodia, lamellipodia, and stress fiber formation in the transfected cells. $\geq 60$ cells were counted for each transfection. $(\mathbf{H})$ The Dbl-shRNA (containing GFP-tag in the pLV-RNAi system) stably transduced SKOV3 cells were stained with Rhodamine-phalloidin and anti- $\alpha$-tubulin plus anti-mouse-AF647. Representative confocal images from three experiments are shown. Decreased actin stress fibers together with lamellipodia changes from the cellular periphery are shown in detail in the magnified area (below). Scale bar, $20 \mu \mathrm{m}$. (I-K) Morphometric analyses of the decreased extent of lamellipodia, actin, and tubulin fibers in Dbl knockdown cells. $\geq 60$ cells were counted for each transfection. (L) Transfected Cos-7 cells were subjected to CyD and NCD treatments before incubation with dextran-AF647 or Tat/pGL3-YOYO-I complexes for uptake. Dbl construct-expressed cells were fixed, permeabilized, and specifically labeled using mouse anti-GST either with anti-mouse-AF488 (the dextran group) or with anti-mouse-AF647 (the Tat/pDNA group), and then subjected to uptake-checking of indicated drugs. Representative confocal images from three experiments are shown. (M, N) The uptake levels of dextran-AF647 and Tat/pGL3-YOYO-I complex in transfected cells were quantified, with $>60$ cells counted per transfection in confocal experiments. Statistically significant differences in relation to the DMSO $(<0.4 \%$, final concentration)-treated control ( $\mathrm{PEBG}-\mathrm{GST}$ ) are shown. $* P<0.05, * * p<0.01$. 
domain-transfected Cos-7 cells were size-enlarged with increased polymerized actin forming a wider cortical actin layer of high stress fiber accumulation (Figure 6C and $\mathrm{E}$ ) and visible lamellipodia (Figure $6 \mathrm{C}$ and $\mathrm{G}$ ). In contrast, the $\mathrm{PH}$ domain-transfected cells showed intermediate levels of cortical actin filament accumulation (Figure 6C and $\mathrm{E}$ ) together with increased filopodia (Figure 6C and F). The lamellipodia in the DH domain expressing cells were similarly increased as those found after $\mathrm{PH}$ domain transfection (Figure 6C and G). Little increased stress fibers, filopodia, and lamellipodia were observed in other domain-transfected cells. Conversely, this onco-Dbl-induced stress fiber and lamellipodia formations were attenuated in SKOV3 cells when Dbl expression was knocked down (Figure 6H-K). Moreover, treatment of onco-Dbl-transfected Cos-7 cells with CyD markedly blocked the uptakes of Tat/pGL3-YOYO-1 and dextran-AF647 to a similar extent (Figure 6L-N), indicating that actin polymerization is essential for onco-Dbl-induced macropinocytosis enhancement. Together, these results indicated that $\mathrm{Dbl}$ expression promotes macropinocytosis through actin cytoskeleton reorganization mainly mediated by the PH domain and Rac1 activation.

\section{Discussion}

Multiple pathways including CvME, CME, and macropinocytosis were reported to mediate the uptake of Tat-delivered nanoparticles, depending on the nature of the cargos/cell interaction..$^{32}$ Nanoparticle effects are mediated not only by its individual components (Tat peptide or pDNA) but also by the formation of nano-size particles, which exert effects of their own. Polyanionic naked pDNA is incapable of internalization, and the highly cationic charged Tat peptide generally uses macropinocytosis for uptake. Therefore, the different transfection efficiencies and varied endocytosis routes exerted by Tat/pDNA nano-complexes (Figure 3E and F) should largely be determined by the particles size with the Tat peptide just helping to condense the cargo DNA. Why medium-size Tat/ pDNA nano-complexes mainly use macropinocytosis for uptake is not well understood. Two possibilities cannot be excluded: 1) the presence of an excess Tat peptide in Tat/ pDNA nano-complexes which have a high cationic charge density stimulates the macropinocytosis pathway. 2) The use of macropinosomes (large sizes, up to $5 \mu \mathrm{m}$ in diameter) rather than clathrin- or caveolae-mediated endosomes (small size, $<100 \mathrm{~nm}$ ) could significantly facilitate the movement of Tat/pDNA nanoparticles inside the cells.

Most of the previous studies emphasized that the physiochemical property of CPP-based nano-complexes affect their uptake. ${ }^{33-35}$ Relatively few investigations proposed that the cell type can also influence the uptake behavior. In the present study, we found that macropinocytosis of the Tat/pDNA nano-complex $(\sim 600 \mathrm{~nm})$ is distinctively high in SKOV3 cells but lower in other cell lines (Figures 4A and C and 5). Macropinocytosis does not frequently operate or occur in nonphagocytic cells. It is only activated or enhanced when specific stimuli (eg, growth factors or viruses) are applied. ${ }^{36}$ Hence, it is likely that the binding of positively charged Tat/pDNA complexes with negatively charged cell surface proteoglycans leads not only to their accumulation on the cell membrane but also to their accelerated macropinocytosis. However, that only SKOV3 cells presented the highest macropinocytosis level among the investigated cells is thought provoking. It was previously found that some CPPs preferentially bind to particular cells whose membrane components favor the specific interaction with CPPs. For example, penetratin peptides were found to be selectively uptaken in neurons because of preferentially binding to $\alpha-2,8$-polysialic acid on the neuronal cell surface. ${ }^{37}$ The anticancer peptide BR2 which targets cancer cells can interact with gangliosides and subsequently is uptaken by lipid-mediated macropinocytosis. ${ }^{38}$ However, most CPPs, such as HIV-Tat, do not have such cell type specificity, because the heparin sulfate or heparin sulfate proteoglycan that they bind to is ubiquitous and redundantly expressed on the surface of all cell types. Therefore, cell-type-specific macropinocytosis variation in this study may probably be due to the tissue origin of the cells. In fact, a study has shown that the uptake mechanism of hydrogel nanoparticles is cell originspecific. ${ }^{39}$ Epithelial cells uniquely internalized nanodiscs and nanorods by the CvME pathway. Human umbilical vein endothelial cells, on the other hand, use the CME pathway for these nanoparticle uptake. ${ }^{39}$ Another interesting study investigated the Tat-pDNA delivery among Cos-7, 3T3, and primary skin fibroblasts. While these cells are all fibroblasts, great transfection variations of Tat-pDNA complexes were observed. ${ }^{16}$ These data collectively point to the importance of cell origin but also strongly reveal some endogenous factors determining the cellular endocytosis phenotype.

Several pieces of evidence support the contribution of oncogenes in macropinocytosis modulation. The original demonstration of $\mathrm{v}$-Src oncogene effects showed that its activation led to a constitutive fluid phase uptake or macropinocytosis in transformed fibroblasts and HeLa cells. ${ }^{10,40,41}$ Another gene-driven macropinocytosis enhancement is the Ras oncogene. H-Ras activation, either through EGF stimulation or due to $\mathrm{G} 12 \mathrm{~V}$ mutation, induced intensive macropinocytosis in HeLa, COS-7, MCF7, and CHO cells. 
Activated K-Ras increased CvME of polyamine in human colon cancer cells, but also promoted the macropinocytosis of extracellular protein or exosomes into pancreatic cells by EGFR stimulation. ${ }^{42-44}$ In the present study, we found that a distinctive onco-Dbl protein exists in SKOV3 cells and that its ectopic expression in Cos-7 cells induces intensive macropinocytosis of Tat/pDNA nano-complexes (Figures 1 and 5). The macropinocytosis promotion was principally contributed through actin cytoskeleton reorganization mainly mediated by the PH domain and Rac1 GTPase activation (Figure 6). Our discovery is consistent with previous reports showing that Rac1-mediated macropinocytosis is responsible for the uptake of DNA-conjugated carbon nanotubes into endothelial cells. ${ }^{10}$ Similarly, previous studies showed the endocytosis regulation function of other Dbl family members. Vav2, one of the GEFs for Rho GTPase, can slow down EGFR CME through its interaction with endosome-associated proteins and requires RhoA activation. ${ }^{40}$ However, Tiam-1, a Racspecific GEF, not only tightly associates with EphA8 but also promotes CME of EphA8-ephrinA5 complexes via Rac activation. ${ }^{41}$ Our results showed that $\mathrm{Dbl}$ expression induced macropinocytosis enhancement but simultaneously inhibited CME (Figure 1). The reason that Dbl and Vav2 inhibited CME whereas Tiam-1 promoted CME may be due to their distinct intra-molecular regulation mechanisms on GEF function exertion. The former two impose active states by PH domain-mediated PI3K (phosphatidylinositol 3-kinase) product [PtdIns $(3,4,5) \mathrm{P} 3]$ binding which seems to alleviate an inhibitory effect from the DH domain, whereas the later may involve an unknown cellular factor which helps maintain the basal GEF state. ${ }^{41}$

Actin-dependent uptake is a feature of macropinocytosis and is further classified as PI3K-dependent or -independent. PI3K-dependent macropinocytosis is linked to Rac1 activation resulting in lamellipodia formation. On the contrary, PI3K-independent macropinocytosis is linked to $\mathrm{Cdc} 42$ activation resulting in filopodia formation. ${ }^{44,45} \mathrm{In}$ the present study, we found that expression of onco-Dbl markedly induced the formation of stress fiber and thereby increased the quantities of lamellipodia (Figure 6). These enhanced actin polymerizations and lamellipodia formation occur alongside macropinocytosis promotion but with the inhibition of CME and CvME (Figure 1), indicating that the macropinocytosis of Tat/pDNA is most likely PI3K-dependent and controlled by the Rac1 activation-mediated actin cytoskeleton rearrangement mechanism. Also, in addition to Rac1 being most significantly activated by onco-Dbl expression, we also found that $\mathrm{Cdc} 42$ and RhoA were activated but not so strongly as
Rac1 (Figure 6A and B). This result is consistent with the aforementioned observation that Rac1 inhibition decreased macropinocytosis more significantly than that by other Rho GTPaeses inhibition (Figure 4). Interestingly, these observations agree with other reports showing that Dbl GEF exhibits broad substrate selectivity but differential catalytic efficiency toward Rho GTPase. ${ }^{30,46}$ Perhaps, the Dbl-induced activation difference to Rac1, Cdc42, and RhoA results from the cellular endocytosis network being shifted to the macropinocytosis pathway, which facilitates a high level of Tat/pDNA uptake in ovarian cancer cells as summarized in Table S4.

\section{Acknowledgments}

This work was supported by the National Natural Science Foundation of China (No 81373318, 30700829), Natural Science Fund of Tianjin (No 16JCYBJC23900), and DoubleFirst Class Fund of Nankai University (No 96163330). We are grateful to Professor Alessandra Eva (Laboratory of Molecular Biology, Istituto Giannina Gaslini, Italy) and Professor Danny Manor (Department of Nutrition, Case Western Reserve University School of Medicine, USA) for kindly supplying the Dbl plasmids. We thank Professor Edward Mignot, Shandong University, for linguistic advice.

\section{Disclosure}

The authors report no conflicts of interest in this work.

\section{References}

1. Wang K, Huang Q, Qiu F, Sui M. Non-viral delivery systems for the application in p53 cancer gene therapy. Curr Med Chem. 2015;22(35): 4118-4136.

2. Lehto T, Kurrikoff K, Langel Ü. Cell-penetrating peptides for the delivery of nucleic acids. Expert Opin Drug Deliv. 2012;9(7):823-836.

3. Kurrikoff K, Gestin M, Langel Ü. Recent in vivo advances in cellpenetrating peptide-assisted drug delivery. Expert Opin Drug Deliv. 2016;13(3):373-387.

4. Eliyahu H, Servel N, Domb AJ, Barenholz Y. Lipoplex-induced hemagglutination: potential involvement in intravenous gene delivery. Gene Ther. 2002;9(13):850-858.

5. Aramaki Y, Takano S, Tsuchiya S. Induction of apoptosis in macrophages by cationic liposomes. FEBS Lett. 1999;460(3):472-476.

6. Cerrato CP, Lehto T, Langel Ü. Peptide-based vectors: recent developments. Biomol Concepts. 2014;5(6):479-488.

7. Wittrup A, Zhang SH, Belting M. Studies of proteoglycan involvement in CPP-mediated delivery. Methods Mol Biol. 2011;683:99-115.

8. Alhakamy NA, Nigatu AS, Berkland CJ, Ramsey JD. Noncovalently associated cell-penetrating peptides for gene delivery applications. Ther Deliv. 2013;4(6):741-757.

9. Margus H, Padari K, Pooga M. Cell-penetrating peptides as versatile vehicles for oligonucleotide delivery. Mol Ther. 2012;20(3):525-533.

10. Zhang B, Wang K, Si J, Sui M, Shen Y. Charge-reversal polymers for biodelivery. In: Gu Z, editors. Bioinspired and Biomimetic Polymer Systems for Drug and Gene Delivery. Weinheim: Wiley-VCH Verlag GmbH \& Co. KGaA; 2014:223-242.

11. Keller AA, Mussbach F, Breitling R, et al. Relationships between cargo, cell penetrating peptides and cell type for uptake of non-covalent complexes into live cells. Pharmaceuticals (Basel). 2013;6(2):184-203. 
12. Wang W, Abbad S, Zhang Z, Wang S, Zhou J, Lv H. Cell-penetrating peptides for cancer-targeting therapy and imaging. Curr Cancer Drug Targets. 2015;15(4):337-351.

13. Jobin ML, Alves ID. On the importance of electrostatic interactions between cell penetrating peptides and membranes: a pathway toward tumor cell selectivity? Biochimie. 2014;107(Pt A):154-159.

14. Macewan SR, Chilkoti A. Harnessing the power of cell-penetrating peptides: activatable carriers for targeting systemic delivery of cancer therapeutics and imaging agents. Wiley Interdiscip Rev Nanomed Nanobiotechnol. 2013;5(1):31-48.

15. Ignatovich IA, Dizhe EB, Pavlotskaya AV, et al. Complexes of plasmid DNA with basic domain 47-57 of the HIV-1 Tat protein are transferred to mammalian cells by endocytosis-mediated pathways. J Biol Chem. 2003;278(43):42625-42636.

16. Hellgren I, Gorman J, Sylvén C. Factors controlling the efficiency of Tat-mediated plasmid DNA transfer. J Drug Target. 2004;12(1): 39-47.

17. Regberg J, Srimanee A, Langel U. Applications of cell-penetrating peptides for tumor targeting and future cancer therapies. Pharmaceuticals (Basel). 2012;5(9):991-1007.

18. Shin MC, Zhang J, Min KA, et al. Cell-penetrating peptides: achievements and challenges in application for cancer treatment. J Biomed Mater Res A. 2014;102(2):575-587.

19. Khalil IA, Kogure K, Futaki S, Harashima H. High density of octaarginine stimulates macropinocytosis leading to efficient intracellular trafficking for gene expression. J Biol Chem. 2006;281(6):3544-3551.

20. Kaplan IM, Wadia JS, Dowdy SF. Cationic TAT peptide transduction domain enters cells by macropinocytosis. J Control Release. 2005; 102(1):247-253.

21. Wadia JS, Stan RV, Dowdy SF. Transducible TAT-HA fusogenic peptide enhances escape of TAT-fusion proteins after lipid raft macropinocytosis. Nat Med. 2004;10(3):310-315.

22. Ognibene M, Vanni C, Segalerba D, et al. The tumor suppressor hamartin enhances Dbl protein transforming activity through interaction with ezrin. J Biol Chem. 2011;286(34):29973-29983.

23. Kauppinen KP, Duan F, Wels JI, Manor D. Regulation of the Dbl protooncogene by heat shock cognate protein 70 (Hsc70). J Biol Chem. 2005; 280(22):21638-21644.

24. Vanni C, Mancini P, Gao Y, et al. Regulation of proto-Dbl by intracellular membrane targeting and protein stability. J Biol Chem. 2002; 277(22):19745-19753.

25. Gao Z, Niu X, Zhang Q, et al. Mitochondria chaperone GRP75 moonlighting as a cell cycle controller to derail endocytosis provides an opportunity for nanomicrosphere intracellular delivery. Oncotarget. 2017; 8(35):58536-58552.

26. Chen H, Gao Z, He C, et al. GRP75 upregulates clathrin-independent endocytosis through actin cytoskeleton reorganization mediated by the concurrent activation of Cdc42 and RhoA. Exp Cell Res. 2016; 343(2):223-236

27. Trabulo S, Cardoso AL, Mano M, de Lima MC. Cell-penetrating peptides-mechanisms of cellular uptake and generation of delivery systems. Pharmaceuticals (Basel). 2010;3(4):961-993.

28. Juliano RL. The delivery of therapeutic oligonucleotides. Nucleic Acids Res. 2016;44(14):6518-6548.

29. Egami Y, Taguchi T, Maekawa M, Arai H, Araki N. Small GTPases and phosphoinositides in the regulatory mechanisms of macropinosome formation and maturation. Front Physiol. 2014;5:374.
30. Lin R, Cerione RA, Manor D. Specific contributions of the small GTPases Rho, Rac, and Cdc42 to Dbl transformation. J Biol Chem. 1999; 274(33):23633-23641.

31. Ueda S, Kataoka T, Satoh T. Role of the Sec14-like domain of Dbl family exchange factors in the regulation of Rho family GTPases in different subcellular sites. Cell Signal. 2004;16(8):899-906.

32. Huang YW, Lee HJ, Tolliver LM, Aronstam RS. Delivery of nucleic acids and nanomaterials by cell-penetrating peptides: opportunities and challenges. Biomed Res Int. 2015;2015:834079.

33. Raucher D, Ryu JS. Cell-penetrating peptides: strategies for anticancer treatment. Trends Mol Med. 2015;21(9):560-570.

34. Murugan K, Choonara YE, Kumar P, Bijukumar D, du Toit LC, Pillay V. Parameters and characteristics governing cellular internalization and trans-barrier trafficking of nanostructures. Int J Nanomedicine. 2015;10: 2191-2206.

35. Singh J, Michel D, Chitanda JM, Verrall RE, Badea I. Evaluation of cellular uptake and intracellular trafficking as determining factors of gene expression for amino acid-substituted gemini surfactant-based DNA nanoparticles. J Nanobiotechnology. 2012;10:7.

36. Mercer J, Helenius A. Virus entry by macropinocytosis. Nat Cell Biol. 2009;11(5):510-520.

37. Bolton SJ, Jones DN, Darker JG, Eggleston DS, Hunter AJ, Walsh FS. Cellular uptake and spread of the cell-permeable peptide penetratin in adult rat brain. Eur J Neurosci. 2000;12(8):2847-2855.

38. Lim KJ, Sung BH, Shin JR, et al. A cancer specific cell-penetrating peptide, BR2, for the efficient delivery of an $\mathrm{scFv}$ into cancer cells. PLoS One. 2013;8(6):e66084.

39. Agarwal R, Singh V, Jurney P, Shi L, Sreenivasan SV, Roy K. Mammalian cells preferentially internalize hydrogel nanodiscs over nanorods and use shape-specific uptake mechanisms. Proc Natl Acad Sci U S A. 2013;110(43):17247-17252.

40. Martin-Perez J, Bar-Zvi D, Branton D, Erikson RL. Transformation by Rous sarcoma virus induces clathrin heavy chain phosphorylation. J Cell Biol. 1989;109(2):577-584.

41. Zhu K, Debreceni B, Bi F, Zheng Y. Oligomerization of DH domain is essential for Dbl-induced transformation. Mol Cell Biol. 2001;21(2): 425-437.

42. Nakase I, Kobayashi NB, Takatani-Nakase T, Yoshida T. Active macropinocytosis induction by stimulation of epidermal growth factor receptor and oncogenic Ras expression potentiates cellular uptake efficacy of exosomes. Sci Rep. 2015;5:10300.

43. Commisso C, Davidson SM, Soydaner-Azeloglu RG, et al. Macropinocytosis of protein is an amino acid supply route in Ras-transformed cells. Nature. 2013;497(7451):633-637.

44. Hawkins PT, Stephens LR. Emerging evidence of signalling roles for PI $(3,4)$ P2 in Class I and II PI3K-regulated pathways. Biochem Soc Trans. 2016;44(1):307-314.

45. Ozdener GB, Bais MV, Trackman PC. Determination of cell uptake pathways for tumor inhibitor lysyl oxidase propeptide. Mol Oncol. 2016;10(1):1-23.

46. Jaiswal M, Dvorsky R, Ahmadian MR. Deciphering the molecular and functional basis of Dbl family proteins: a novel systematic approach toward classification of selective activation of the Rho family proteins. J Biol Chem. 2013;288(6):4486-4500. 


\section{Supplementary materials}

Table SI Amount of HIV-Tat necessary to form Tat/pGL3-YOYO-I complexes (10 $\mu \mathrm{g}$ PDNA/mL)

\begin{tabular}{lllll}
\hline N/P ratio & $1: 1$ & $5: 1$ & $10: 1$ & $20: 1$ \\
{$[$ Tat $], \mu M$} & 3.44 & 17.11 & 34.19 & 68.38 \\
\hline
\end{tabular}

Notes: N/P ratio is the molar ratio of total free amino groups (positive charge) in Tat peptide to total free phosphate groups (negative charge) in pDNA taken in solution The detailed calculation was described in Damodaran and Murthy' and Caputo et al. ${ }^{2}$

Table S2 Concentration of endocytosis inhibitors and markers used in this study

\begin{tabular}{|c|c|c|c|}
\hline Drug/treatment & $\begin{array}{l}\text { Final } \\
\text { concentration }\end{array}$ & Pathway targeted/marked & Mode of action \\
\hline $4^{\circ} \mathrm{C}$ & & All endocytosis & Inhibition of energy-dependent processes \\
\hline Sodium azide & $0.1 \%, \mathrm{~m} / \mathrm{v}$ & All endocytosis & Metabolic inhibitor \\
\hline Chloroquine & $300 \mu \mathrm{M}$ & CME & $\begin{array}{l}\text { Affects the function of CCVs. Inhibits acidification of } \\
\text { endosomes }\end{array}$ \\
\hline Chlorpromazine (CPZ) & $10 \mu \mathrm{g} / \mathrm{mL}$ & CME & $\begin{array}{l}\text { Translocates clathrin and AP2 from the cell surface } \\
\text { to intracellular endosomes. Inhibits } \mathrm{CIE} \text { in some cells }\end{array}$ \\
\hline Cytochalasin D (CyD) & $5 \mu \mathrm{M}$ & Most endocytosis & Depolymerizes F-actin \\
\hline Nocodazole (NCD) & $10 \mu \mathrm{M}$ & Vesicle trafficking & Disrupt microtubule network \\
\hline Methyl-b-cyclodextrin (MBCD) & $\mathrm{I} \mathrm{mM}$ & Lipid raft, CME, fluid phase endocytosis & Removes cholesterol from the plasma membrane \\
\hline Filipin & $5 \mu \mathrm{g} / \mathrm{mL}$ & CIE, CrME & $\begin{array}{l}\text { Sterol-binding agent, disrupts caveolar structure and } \\
\text { function. Binds to cholesterol in the membrane }\end{array}$ \\
\hline $\begin{array}{l}\text { 5-(N-Ethyl-N-isopropyi)- } \\
\text { amiloride (EIPA) }\end{array}$ & $10 \mu \mathrm{M}$ & Macropinocytosis & Inhibits $\mathrm{Na}+/ \mathrm{H}+$ exchange; may affect actin \\
\hline Tfn-AF647 & $25 \mu \mathrm{g} / \mathrm{mL}$ & CME & \\
\hline Dextran-AF647; dextran-RITC & $4 \mathrm{mg} / \mathrm{mL}$ & Macropinocytosis & \\
\hline CTxB-AF647 & $10 \mu \mathrm{g} / \mathrm{mL}$ & CIE, CvME & \\
\hline
\end{tabular}

Abbreviations: CIE, clathrin-independent endocytosis; CCV, clathrin-coated vesicle; CME, clathrin-mediated endocytosis; CvME, caveolae-mediated endocytosis.

Table S3 Dbl gene knockdown sequences used in this study

\begin{tabular}{|c|c|c|}
\hline & Targeting sequence (mRNA: $\left.5^{\prime}-3^{\prime}\right)$ & $\begin{array}{l}\text { Efficiency } \\
\text { (protein level), \% }\end{array}$ \\
\hline \multirow[t]{3}{*}{ siRNA duplexes ${ }^{\#}$} & GGAAGAAGTTTATATTGTC (2349-2367) & $70-85$ \\
\hline & GGTGATAACCGCAAGTTTG (23| I-2329) & $65-90$ \\
\hline & GGAGCAGTATCTGCAACTA $(642-660)$ & $50-60$ \\
\hline shRNA (pLV-GFP- & GGGACTATAGCTCAAGTAA (745-763) & $35-45$ \\
\hline \multirow[t]{2}{*}{ RNAi system) ${ }^{\#}$} & GGGCAAGATGATAATGCAA (2073-209I) & $50-65$ \\
\hline & GCAGAAGCAAATACAGTTTG $(26 \mid 7-2636)$ & $75-90$ \\
\hline
\end{tabular}

Note: "The sequences in bold were finally used for knockdown approaches as described in the "Materials and methods" section. 


\section{Tat/pGL3-YOYO-1}

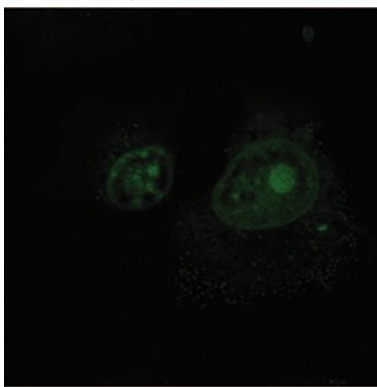

Tat/pGL3-YOYO-1

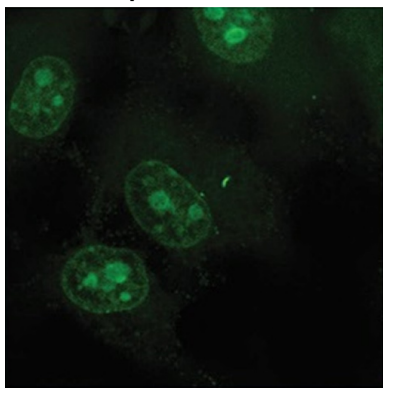

Tfn-AF647

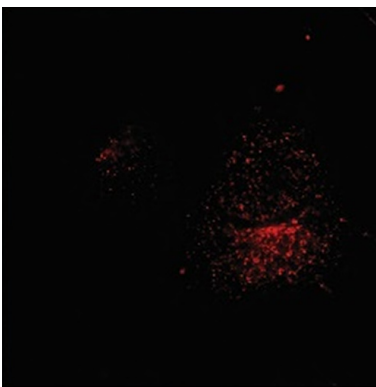

CTxB-AF647

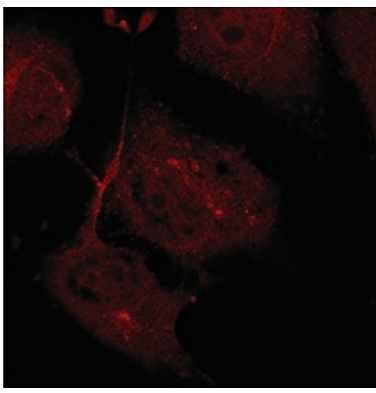

Merge

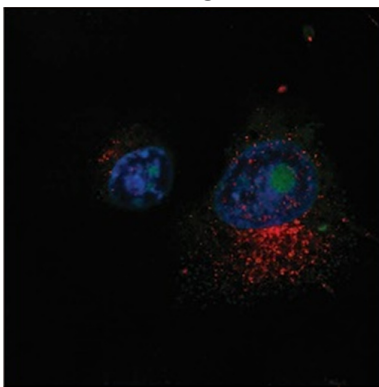

Merge

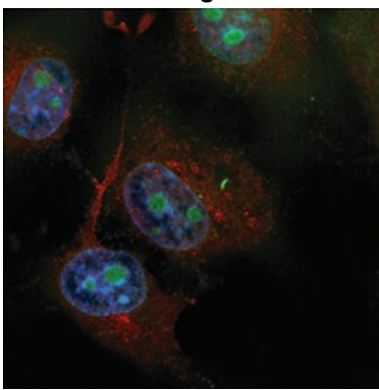

Figure SI Confocal imaging analyzed the uptake of Tat/pDNA together with endocytosis markers.

Notes: Confocal imaging analysis of the co-localization of Tat/pGL3-YOYO-I with CME and CvME markers. COS-7 cells were co-incubated by Tat/pGL3-YOYO-I complexes (N/P=10, green emission signal) together with dextran-AF647 and CTxB-AF647 (red emission signal) for I hour. Cell nuclei were stained with Hoest33I2 (blue emission signal).

Abbreviations: CME, clathrin-mediated endocytosis; CvME, caveolae-mediated endocytosis.

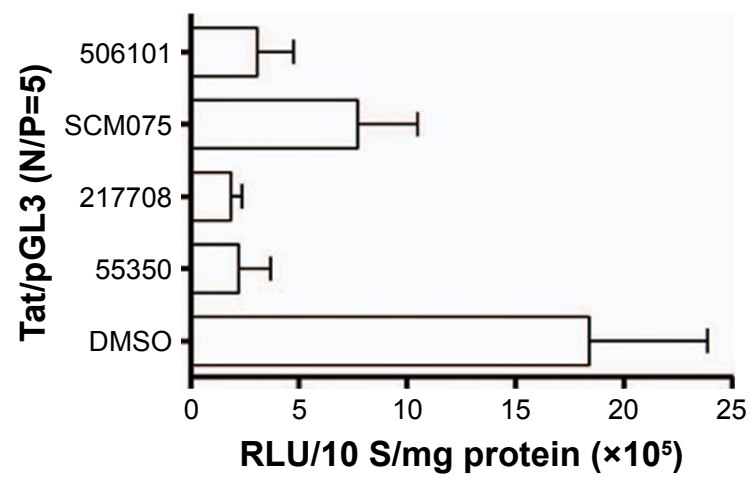

Figure S2 Luciferase expression analysis of Tat/PGL3 transfection with Rho GTPase inhibitors.

Note: Luciferase activity was determined in Tat/pGL3 (N/P=5) transfected SKOV3 cells, which were treated with Rho GTPase inhibitors as described in the "Materials and methods" section.

A

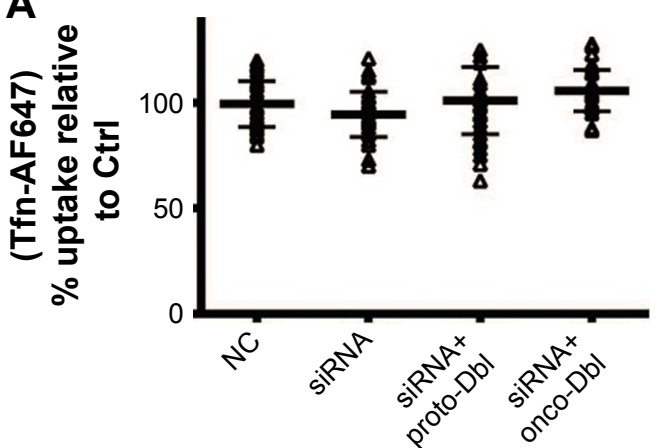

B

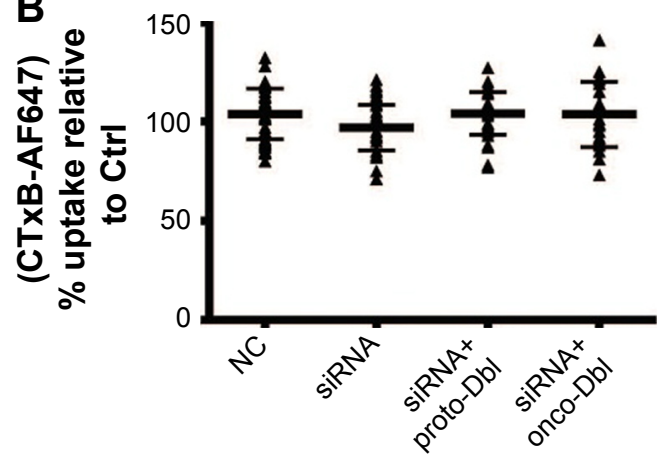

Figure S3 Confocal imaging analyzed the uptake of (A) Tfn-AF647 and (B) CTxB-AF647 after endogenous Dbl knockdown followed by Dbl-GST overexpression.

Notes: Scatterplots depict the uptake changes of indicated drugs in single-cell populations. Uptake levels in non-transfected SKOV 3 cells were set at $100 \%$ for comparison. NC, scb-siRNA transfected SKOV3 cells. $\geq 60$ cells were counted for each transfection. 
Table S4 Summary of Dbl's modulation activity

\begin{tabular}{|c|c|c|c|c|c|c|c|}
\hline & $\begin{array}{l}\text { Tat/pDNA } \\
\text { uptake }\end{array}$ & $\begin{array}{l}\text { Macropinocytosis } \\
\text { (Dextran) }\end{array}$ & $\begin{array}{l}\text { CME } \\
\text { (Tff) }\end{array}$ & $\begin{array}{l}\text { CvME } \\
\text { (CTxB) }\end{array}$ & $\begin{array}{l}\text { Racl } \\
\text { activation }\end{array}$ & $\begin{array}{l}\text { Cdc42 } \\
\text { activation }\end{array}$ & $\begin{array}{l}\text { RhoA } \\
\text { activation }\end{array}$ \\
\hline Proto-Dbl (I-925) & ++ & ++ & - & - & + & NC & NC \\
\hline Onco (498-925) & ++++ & ++++ & - & - & ++++ & +++ & +++ \\
\hline DH-PH (498-825) & ++++ & +++ & -- & NC & +++ & ++ & + \\
\hline TI (I-825) & ++ & +++ & - & - & & & \\
\hline$N(I-498)$ & NC & $\mathrm{NC}$ & NC & NC & & & \\
\hline SP (224-4I7) & $\mathrm{NC}$ & $\mathrm{NC}$ & NC & NC & & & \\
\hline PH (7II-808) & ++++ & +++ & - & - & +++ & ++ & + \\
\hline $\mathrm{DH}(498-703)$ & ++ & NC & NC & NC & ++ & NC & $\mathrm{NC}$ \\
\hline DH-PH 7M (498-724) & $\mathrm{NC}$ & $\mathrm{NC}$ & NC & NC & & & \\
\hline DH-PH VI (498-7I0) & NC & $\mathrm{NC}$ & NC & NC & & & \\
\hline
\end{tabular}

Notes: Proto-Dbl comprises different domains that might be involved in endocytosis regulation, Rho GTPase activation, and Tat/pDNA delivery. +, promotion; -, inhibition.

Abbreviations: CME, clathrin-mediated endocytosis; CVME, caveolae-mediated endocytosis; NC, no change.

\section{References}

1. Damodaran VB, Murthy NS. Bio-inspired strategies for designing antifouling biomaterials. Biomaterials Research. 2016;20(18):1-11.
2. Caputo A, Betti M, Altavilla G, et al. Micellar-type complexes of tailormade synthetic block copolymers containing the HIV-1 tat DNA for vaccine application. Vaccine. 2002;20(17-18):2303-2317.
International Journal of Nanomedicine

\section{Publish your work in this journal}

The International Journal of Nanomedicine is an international, peerreviewed journal focusing on the application of nanotechnology in diagnostics, therapeutics, and drug delivery systems throughout the biomedical field. This journal is indexed on PubMed Central, MedLine, CAS, SciSearch ${ }^{\circledR}$, Current Contents ${ }^{\circledR} /$ Clinical Medicine,

\section{Dovepress}

Journal Citation Reports/Science Edition, EMBase, Scopus and the Elsevier Bibliographic databases. The manuscript management system is completely online and includes a very quick and fair peer-review system, which is all easy to use. Visit http://www.dovepress.com/ testimonials.php to read real quotes from published authors. 